\title{
ORIGINE DE L'UTILISATION PRIVILÉGIÉE DU TERME DE $A M R$ CHEZ LES MU'MINIDES ALMOHADES
}

\author{
ÉMILE FRICAUD \\ Vernon, Francia
}

Dans les textes qui nous sont parvenus, composés durant le long siècle des Mu'minides almohades, c'est-à-dire pendant les règnes de leurs douze califes -d'Abd al-Mu'min à Abū Dabbūs-, il est facile à tout lecteur attentif de discerner une utilisation privilégiée du terme de $a m r$ pour désigner soit un «ordre donné», soit «l'autorité» califale et, finalement, la personne-même du calife qui devient alors «l'Autorité».

Comme il ne saurait être question ici de nous engager dans un examen exhaustif de la totalité de ces textes retrouvés pour y extraire tous les exemples qui y sont attestés de cette utilisation spécifique du mot $a m r$, je voudrais simplement tenter un tel relevé dans l'un des plus «précieux» de ces textes: le fragment édité du Ta'rịh al-mann d' Ibn Șāhịib aș-Șalāt ${ }^{1}$, en ne recourant que pour confirmation à quelques autres écrits disponibles. Je me permettrai ensuite de suggérer que soit reconnue l'origine de cette utilisation mu'minide du mot $a m r$ dans une exclamation fameuse du mahdi Ibn Tümart concernant ${ }^{\mathrm{c}} \mathrm{Abd}$ al-Mu'min dont je pense qu'elle a été jusqu'à présent mal comprise par les «orientalistes» maghribistes et à laquelle il convient donc, selon moi, de redonner sa vraie signification.

\section{Le mot amr dans la langue arabe}

Le mot amr se présente en arabe sous la forme de deux homonymes: amr (plur. umūr) qui signifie «chose», «affaire», «état de cho-

\footnotetext{
${ }^{1}$ Kitāb Ta'rīh al-mann bi-l-imāma 'alä-l-mustad̆afin bi-an ğacala-hum Alläh a'immat wa-ğa ala-hum al-wäriținn... «Histoire du don de l'Imāmat fait à des hommes considérés comme faibles mais que Dieu a constitués imām(s) et établis comme héritiers". (Qur'ān, VII, 137 et XXVIII, 5) Bodléienne d'Oxford, Ms. unicum. Mes références (T.m.) vont à la $1^{\text {ère }}$ édition d'c Abd-al-Hādī At-Tāzī, Beyrouth, 1964.
}

Al-Qanțara XXIII, 1 (2002) 93-121 
se», «situation», et amr (plur. awāmir) qui signifie «ordre», «commandement», «autorité». C'est dans ce second sens que le mot va ici nous intéresser, mais sans que le premier soit à perdre de vue puisqu'il va interférer dans la remise en cause que je vais proposer de l'interprétation donnée jusqu'ici à la dite parole historique prononcée par Ibn Tūmart.

\section{Le mot amr dans la terminologie islamique}

Présent un grand nombre de fois dans le Qur'ān ${ }^{2}$ pour désigner 1' Ordre péremptoire divin qui déclenche l'action, ce terme de $a m r$ fut, semble-t-il, tôt perçu en Islām, comme d'utilisation délicate. Seul Dieu détient l'Autorité radicale, c'est-à-dire la capacité de donner l'ordre qui produit l'être ou provoque l'agir. A la question d'une participation humaine ontologique à cette Autorité, le texte sacré donne une réponse négative dans le verset 154 de la sourate III ( $\left.A l-^{c} \operatorname{Imrān}\right)$ :

«... ils demandent: 'avons-nous droit de participation de quelque façon au commandement?'; réponds-leur que le commandement tout entier est à Dieu! (inna-l-amr kulla-hu li-Alläh!)» ${ }^{3}$.

et il est à noter que cette déclaration sans appel est un hapax puisque formulée une seule fois dans le saint livre. Quant à l'autorité détenue par les souverains, elle a son principe en Dieu même et l'homme ne peut être que l'exécuteur du «commandement» divin. C'est le sens du verset 123 de la sourate XI $(H \bar{u} d)$ :

«... à Lui s'origine tout ce qui est commandement! (wa-ilay-hi yurğa $a^{c} u$ l-amr kullu-hu)» ${ }^{4}$.

\footnotetext{
2 On peut voir en E.I. ${ }^{2}, \mathrm{I}$, l'article «Amr» de S. Pines. Si l'on consulte l'Al-Mu ğam al-mufahras (Dictionnaire des mots du Qur'ān), de Muhammad Fu'ād 'Abd-al-Bāqī, on trouve, pour le mot $a m r$, cinq longues colonnes de références. Il est à remarquer que dans les traductions en langues européennes des versets où apparaît ce mot, il y a souvent hésitation entre les deux sens: de «chose», «affaire» et d' «ordre», «commandement».

${ }^{3}$ Denise Masson, dans sa traduction, prend ici le mot $a m r$ dans son sens d' «affaire»: Ils disaient: «Y a-t-il quoi que ce soit qui nous concerne dans cette affaire?». Dis: «L'affaire tout entière appartient à Dieu!». Jacques Berque, lui, traduit: «N'avons-nous, disaient-ils, quelque part au décret?»... Dis: «Le décret revient à Dieu dans sa totalité».

4 Traduction Denise Masson: «Toute chose revient à Lui». Traduction Berque: «De Lui relève l'intégralité du décret».
} 


\section{Le mot amr chez les almohades}

\section{Sur les monnaies almohades}

Il apparaît que les Mu'minides almohades eurent une conscience particulièrement aiguë de cette valeur qur'ānique du mot $a m r$. En témoignent les formules inscrites sur leurs monnaies: $\operatorname{dirham}(s)$, qirāțts) (demi dirham), et $\operatorname{din} \overline{a r}(s)^{5}$. Les $\operatorname{dirham}(s)$ carrés, en argent, dont plusieurs milliers ont été retrouvés, de même que les qirāt $(s)$, portent tous, côté revers, en deuxième ligne, la formule du verset ci-dessus (Q. III - 154): «al-amr kullu-hu li-Alläh (Le Commandement tout entier est à Dieu)» alors que les $\operatorname{din} \bar{r} r(s)$ d'or, ronds, portent, à l'intérieur du carré inscrit dans le cercle, côté avers, la formule: «al-qā'im bi-amr Allāh (L'exécuteur du Commandement de Dieu)» pour désigner d'abord le mahdī Ibn Tümart, plus tard, son «lieutenant» (haliffa) 'Abd al-Mu'min et, ensuite, chacun de ses successeurs; formule que l'on retrouve régulièrement attestée dans la tardiya (appel de l' "agrément» divin sur la personne du calife) des lettres almohades ${ }^{6}$. Dieu seul est détenteur du amr; le chef de la communauté n'est que l'exécuteur de ce $a m r$ divin. Telles sont les affirmations exprimées par le Qur'ān et gravées sur les monnaies almohades.

\footnotetext{
5 Sur les découvertes de monnaies almohades, on peut consulter, entre autres publications: Bel, A., «Contribution à l'étude des dirhems de l'époque almohade d'après un groupe important de ces monnaies récemment découvert à Tlemcen», Hespéris, 16 (1933), 1-18 (L'étude porte sur 3800 dirham(s) almohades trouvés dans une jarre en 1932); «Hallazgos numismáticos musulmanes», Al-Andalus, XII (1947), 481-84 (L'étude porte sur des pièces, dont certaines almohades, trouvées en 1946 à Nisa, au Portugal); Eustache, D., «Al-Basra, capitale idrissite et son port», Hespéris, XLII (1955), 217-38; en appendice n. ${ }^{\circ} 3$, p. 236-38: étude d'une pièce d'argent, un qirāt carré; Guastavino Gallent, G., «Notas de numismática magrebí», Tamuda, III (1955), 116-21 (L'étude porte sur 223 dirham(s) almohades découverts en 1954, au Maroc, près de Cabo de Agua ( $R a$ 's $a l-m \bar{a}$ '); Esteve Guerrero, M., «Hallazgo de dirhemes almohades en Jerez de la Frontera», Al-Andalus, XXVI (1961), 227-28; Bourouiba, R., "Monnaies et bijoux trouvés à la Qal'a des Banī Hammād", Revue de l'Occident musulman et de la Méditerranée, 8 (1970), $67-77$ (L'étude porte sur plus de 1000 dirham(s) et 18 dīnār(s) almohades trouvés en 1967); «Sur six dīnārs almohades trouvés à la Qal'a des Banī-Hammād», Bulletin d'archéologie algérienne, II (1966-1967), 271-291; Bourouiba, R., 'Abd-al-Mu'min flambeau des Almohades, Alger, 1974, 77-79; Musées d'Algérie, n. ${ }^{\circ}$ 1, S.N.E.D., 1971, p. 83.

6 Sur les lettres almohades, voir Fricaud, É., «Les talaba dans la société almohade», Al-Qanțara, XVIII-2 (1997), p. 334, note 9.
} 


\section{Dans les textes mu'minides}

Forts de cette idée que leur "autorité» leur était conférée par Dieu lui-même, les califes mu'minides en arrivèrent à faire du mot $a m r$ un usage dynastique en insistant pour qu'à côté de leurs titres de "Calife» (halïfa), de "Commandeurs des croyants» (Amïr al-mu'minin) ${ }^{7}$, d' «Imām» de la communauté et de suprême Sayyid, leur soit reconnue de façon originale leur qualité d' exécuteur du amr divin par attribution de l'appellation personnifiée d'al-Amr («l'Autorité»). De cette originalité, les textes retrouvés, écrits sous leurs règnes, font foi, en particulier donc le fragment retrouvé et édité du Ta'rīh al-mann d' Ibn Șāhib aṣ-Șalāt (2 ème sifr d'un ouvrage qui en comptait trois). Dans ces textes, le mot amr (plur. awämir), employé quelquefois seul mais le plus souvent renforcé d'un qualificatif épithète, y désigne soit l'ordre donné, soit «l'Autorité», c'est-à-dire le pouvoir califal et le Calife en personne. Sont ainsi attestées, avec l'une ou l'autre de ces deux significations, les formulations suivantes ${ }^{8}$ :

\footnotetext{
- al-amr ......... «l'ordre». . . . . . ou . . «l'Autorité »

- hãda $l$-amr .................... «cette Autorité»

—al-amr al - ${ }^{c} a z i z$. . . . . . «l'ordre insigne». . . ou . . «l'Autorité toute-puissante»

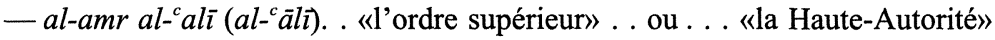

— al-amr al-karim. . . . . «l'ordre noble». . . . ou . . «la Noble-Autorité»

— al-amr al-mutăa $\ldots \ldots \ldots$. . . . . . . . . . «l'Autorité-Obéie»

- al-amr as-sacid. . . . . . . . . . . . . . . . . «l'Heureuse-Autorité»

—al-amr al-cazìm . . . . . . . . . . . . . «la Sublime-Autorité»

- hā
}

Dans le sens d'«ordre donné», d'«ordre reçu»

Dans ce sens, le mot se trouve utilisé soit au singulier (al-amr) soit au pluriel (al-awāmir). Voici quelques exemples, un par formulation attestée ${ }^{9}$ :

\footnotetext{
7 Dans $a m i \tilde{r}$ il y a $a m r$.

8 Les formulations données ici sont celles attestées dans les textes utilisés par moi; il est vraisemblable que d'autres variantes (avec d'autres «qualificatifs») apparaissent dans d'autres textes.

${ }^{9}$ En plus de la référence à l'exemple donné, je note, éventuellement, les références à d'autres exemples semblables.
} 
- al-amr: «l'ordre», «l'autorité» - al-awāmir: «les décisions»

- Il est dit qu'après la victoire des Almohades à Grenade en 557/ $1162,{ }^{\circ} \mathrm{Abd}$ al-Mu'min décida que Cordoue deviendrait «siège de l'autorité» (istiqrār al-amr) et "siège des décisions» (istiqrār al-awämir) [T.m., p. 203, 1.4 et 1.6].

—al-amr al- ${ }^{c} a z \bar{z}:$ «l'ordre insigne» - al-awāmir al- ${ }^{c}$ azizza: «les insignes instructions»

- Il est dit que les deux sayyid(s), Abū-Ya ${ }^{c} q u \bar{b}$ et $\mathrm{Abū}-\mathrm{Sa}^{\mathrm{c}} \overline{\mathrm{i}} \mathrm{d}$, avaient reçu «l'ordre insigne» de s'établir à Cordoue [T.m. p. 201, 1. 17]; comme il est parlé des «insignes instructions» qui furent données par le calife Abū Ya ${ }^{c} q u ̄ b$ à son arrivée à Séville en 566/1171 [T.m. p. 453, 1.1] [Autres exemples: id., p. 172, 1.1; p. 199, 1.16; p. 217, 1.11; p. $231,1.14$; p. $297,1.2$; p. $353,1.7$; p. 383 , 1.7; p. $429,1.16]$.

— al-amr al- ${ }^{c} a l i \bar{\imath}\left(a l-^{c} \bar{a} l \bar{l}\right)$ : «l'ordre supérieur» - al-awāmir al- ${ }^{c} a \bar{l} l y y a$ (al-caliyya): «les instructions supérieures»

- Il est dit qu'en 568/1172, de retour de l'expédition de Huete, le calife $\mathrm{Abū} \mathrm{Ya}^{\mathrm{c}} \mathrm{qu} \mathrm{u}$ entra à Séville et qu'alors fut confirmé son «ordre supérieur» de construire la grande mosquée [T.m. p. 517, 1.5]; comme il avait été parlé de la lettre circulaire qui avait été expédiée en l'année 562/1167 à tout le pays et qui avait constitué «la première de ses instructions supérieures» (awwal awämir al- $\left.{ }^{c} a ̈ l i y a\right)$ [T.m., p. 302, 1.13]. [Autre exemples: id. p. 233, 1.16-17; p. 302, 1.6].

- al-amr al-karim: «l'ordre noble»

- Il est dit qu'en l'année 557/1162, après leur victoire de Grenade, les deux sayyid(s), $A b \bar{u} \mathrm{Ya}^{c}$ qūb et $\mathrm{Abū} \mathrm{Sa}{ }^{c} \bar{i} d$, allèrent s'installer à Cordoue conformément à «l'ordre noble» reçu d' 'Abd al-Mu'min [T.m., p. 205, 1.6] [Autres exemples: id., p. 257, 1.4; p. 522, 1.10; p. 526, 1.3]. ${ }^{10}$

10 On sait que lorsqu'il composa son Bayān almohade (B. alm., éd. Casablanca, 1985) [ ${ }^{\text {ème }}$ partie de l'Al-Bayān al-mugirib], après la disparition des Almohades, au début du VIII/XIV ème siècle, sous les Mérinides, Ibn 'Idārī utilisa le T.m. d'Ibn Șāḥib aș-Ṣalāt en en "désalmohadisant» passablement le texte. Il me paraît intéressant ici d'indiquer ce que sont devenues dans le Bayān les formules que nous venons d'examiner, lorsqu'elles se trouvaient dans des passages repris par Ibn 'Id̄āri. Remarquons que, dans ces formules, 
Dans le sens de "l'Autorité» pour désigner le calife régnant en personne

Employé alors uniquement au singulier, le mot al-Amr «l'Autorité», ainsi personnifié (et éventuellement solennisé sous la forme «hād $\bar{a}-l-A m r »$ «cette Autorité»), se retrouve soit seul, soit, le plus souvent, renforcé par des épithètes encore plus variées que dans le premier usage.

\section{- al-Amr: «l'Autorité»}

- Il est dit qu'en 561/1165, au retour du siège de Murcia, le sayyid $A b u ̄ S^{c} \bar{i} d$ s'installa à Cordoue «à la suite d'un avis de l'Autorité [... bi-ra'y min al-Amr...] (c'est-à-dire du calife $A b u \bar{~} \mathrm{Ya}^{\mathrm{c}} \mathrm{qu} \mathrm{u}$ ) précédemment exprimé» [T.m., p. 287, 1.1].

- Il est dit que «l'Autorité» [al-Amr] (c'est-à-dire le calife Abū $\mathrm{Ya}^{\mathrm{c}} \mathrm{qu} \mathrm{u}$ ) trouva excellente une qașída (poème) déclamée devant lui pour chanter une victoire remportée sur des dissidents [T.m., p. 367, 1.5].

- Il est dit que Fernando Rodríguez, après un séjour à Marrakech, faillit embrasser l'Islām, et s'engagea à travailler au service des musulmans «sur le conseil de l' Autorité [... fi nașhi l-Amr]» (c.-à-d. du

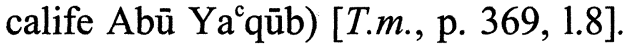

l'emploi du mot amr (plur. awāmir) dans son sens d'«ordre» d' (instructions» n'avait rien d'hétérodoxe; seule la présence redondante des épithètes pouvait irriter l'auteur du Bayān. Celui-ci a parfois retenu intégralement les formules; ainsi en est-il de celle d'al-amr al- ${ }^{c} a z \bar{z} z$ attestée en B. alm.,p. 77, 1.11 (copiée en T.m., p. 199, 1.16) et en B. alm., p. 107, 1.3-4 (copiée en T.m., p. 383, 1.7) et de celle d' al-awämir al-'aliyya deux fois retenue dans la même page en $B$. alm., p. 94, 1.17 et 20 (copiée en T.m.,p. 302,1.6 et 13). Mais d'autres fois —et c'est à noter - Ibn 'Idārī a retenu «l'ordre» (al-amr) donné, mais en omettant l'épithète de redondance: en $B$. alm., p. $77,1.19$ on lit que «l'ordre» arriva aux deux sayyid(s) d'avoir à s'établir à Cordoue, alors qu'en T.m., p. 203, 1.8 il y avait «l'ordre insigne»; en $B$. alm., p. 93, 1.17, on lit qu'arriva au šayh Ibn Abī Ibrāhīm «l'ordre» d'aller assurer le gouvernorat de Grenade, alors qu'en T.m., p. 297, 1.2 il y avait «l'ordre insigne»; en $B$. alm., p. 101, 1.3, on lit que l'annonce d'une baraka plénière de la part du calife Abū Ya qūb fut transmise «par son ordre» à ses frères gouverneurs, alors qu'en T.m., p. 353, 1.7 il y avait «par son ordre insigne»; en $B$. alm., p. 86, 1.14-15, on lit, à propos du sayyid Abū Hafs, qu'on appliqua «son ordre» de repartir, alors qu'en T.m., p. 257, 1.3-4, il y avait «son ordre noble». Il arrive aussi qu'une tournure neutre soit substituée par Ibn 'Idāāi à la formu-

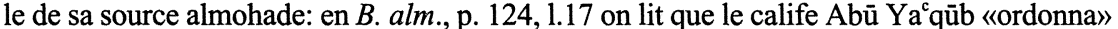
que fût bâtie la Grande Mosquée (de Séville) [... amara bi-binā' al-ǧāmic $]$, alors qu'en T.m., p. 517, 1.5, il y avait: 'fut confirmé son ordre supérieur de construire la Grande Mosquée' [... ta'akkada amru-hu al-'álí fi binä' al-ğāmic] 
- Il est dit qu'après que des fonctionnaires de Séville eurent calculé le prix de dédommagement des maisons à démolir pour permettre l'aménagement du quartier de la nouvelle Grande Mosquée, «l'Autorité entérina leur estimation [... fa-amḍa al-Amr taqdira-hum]». Il s'agit ici du calife Abū Yūsuf Ya qūb al-Manșūr [T.m., p. 485, 1.9].

- Il est rappelé que le šayh Abū Hafș «avait autrefois conseillé 1'Autorité [wa-huwa alladī nasaha al-Amr qadìman]», c.-à-d. le calife $\mathrm{Abū} \mathrm{Ya}{ }^{\mathrm{c}} q u \bar{b}$ [T.m., p. 524, 1.4].

- la variante $h \bar{a} \underline{d} \bar{a}-l-A m r:$ «cette Autorité»

Cette variante, sans épithète, n'est pas attestée - sauf inadvertance de ma part- dans notre fragment retrouvé du Ta'rīh al-mann. On la trouve, par contre, utilisée par Abū l-Hasan ${ }^{c} \mathrm{Alī} \mathrm{Ibn} \mathrm{al-Qațtān} \mathrm{(Ibn}$ al-Qațtān père) dan son récit ${ }^{11}$ concernant un jeune visionnaire illuminé, fils d'un țālib almohade, dont il fut chargé d'élucider le cas en sa qualité de šayḥ des tālib(s)-de-la-présence.

- Nous y entendons le père de ce garçon qui, après avoir fait assister son fils à une impressionnante revue des forces armées califales, lui demande: «Mon fils, as-tu vu ce que Dieu - exalté soit-Il! — a accordé de puissance aux hommes de Cette Autorité (ahl hād $\bar{a}-l-A m r)$ ?» (Il s'agit du calife de l'époque: al-Mustanșir) [Dayl-8, p. 183, 1.19].

- Ibn al-Qatțān raconte ensuite comment il dut informer ce père et son fils qu'ils auraient à comparaître devant les hommes de l'Autorité (ahl al-Amr) [Dayl-8 p. 187, 1.22; p. 188, 1.14; p. 189, 1.19].

- al-Amr al- ${ }^{c} a z i \bar{z}:$ «l'Autorité toute-puissante»

— variante: $h \bar{a} d \bar{a}-l-A m r$ al- ${ }^{c} a z i \bar{z}:$ «cette Autorité toute-puissante»

- Il est parlé du wālī («gouverneur») de Cordoue, Abū Zayd Ibn Tīğit qui, en 554/1159, opposa à Ibn Mardanīs la résistance des «hommes fidèles, en tout état de cause, au serment prêté à l'Autorité toute-puissante (c.-à-d. au calife 'Abd al-Mu'min) [... ahl al-wafä' bi-l-bï a li-l-Amr al- ${ }^{c} a z \bar{\imath} z$ fi kulli hāal]» [T.m., p. 116, 1.7].

\footnotetext{
${ }^{11}$ Ce récit autobiographique est rapporté par Ibn ${ }^{\mathrm{c}} \mathrm{Abd}$-al-Malik al-Marrākušĩ dans la notice qu'il consacre à Abū l-Ḥasan Ibn al-Qatțān dans l'Ad-Dayl wa-t-Takmila [8 $8^{\text {ème }}$ sifr, éd. M. Bencherifa, Rabat, Académie Royale Marocaine, 1984, 165-95, notice n. ${ }^{\circ} 10$. Réf. ici: «Dayl-8». J'ai donné une traduction française de cette notice dans les Biografias almohades. II, Estudios onomástico-biográficos de al-Andalus, X, ed. M. L. Ávila y M. Fierro, Madrid-Granada, 2000, 223- 283.
} 
- Il est dit qu'au temps où $\mathrm{Abū} \mathrm{Ya} \mathrm{a}^{\mathrm{c} q u ̄ b}$ était wālī («gouverneur») de Séville, les dignitaires de cette ville étaient «tout dévoués à cette Autorité toute-puissante (c.-à-d. au calife ${ }^{\mathrm{C}} \mathrm{Abd}$ al-Mu'min) [al-muhlișin li-hādā-l-Amr al- $\left.{ }^{c} a z \bar{z}\right]$ ]» [T.m., p. 120, 1.2].

- Il est dit que, joint à la lettre expédiée par ${ }^{\mathrm{C}} \mathrm{Abd}$ al-Mu'min depuis l'Ifrīqiya à son fils $\mathrm{Abū} \mathrm{Ya}^{\mathrm{c}} \mathrm{qu} \mathrm{b}$ à Séville, il y avait un morceau poétique que lui, Abū Ya ${ }^{c} q u ̄ b$, prétendit être «de la plume de l'Autorité toute-puissante (c.-à-d. du calife ${ }^{\mathrm{c}} \mathrm{Abd}$ al-Mu'min) [... min

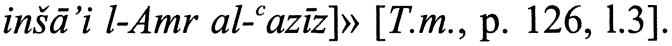

- Il est dit plusieurs fois que tel ou tel évènement se produisit «de par la bonne fortune de (cette) Autorité toute-puissante [... $b i$-sa $a^{c} d$ (hāda $)$ l-Amr al- $\left.{ }^{c} a z \bar{z}\right]$ ]» ou «de par la baraka de (cette) Autorité toute-puissante [... bi-baraka (häd̄a) l-Amr al- $\left.{ }^{c} a z \bar{i}\right]$ ]» [T.m., p. 130, 1.4; p. $181,1.16$; p. $187,1.9 ;$ p. $388,1.6$; p. $400,1.22]$.

- Il est dit que lors du passage d' 'Abd al-Mu'min en al-Andalus après sa campagne d'Ifrīqiya en 555/1160, son fils, Abū Ya ${ }^{\mathrm{c}} \mathrm{qu} \overline{\mathrm{b}}$, wāli («gouverneur») de Séville, aurait voulu pouvoir devancer tout le monde «pour rencontrer l'Autorité toute-puissante [... fi liqa'̄' $\mathrm{l}$ l-Amr al- $\left.{ }^{c} a z \overline{i z}\right] »$ [T.m., p. 145, 1.1].

- Il est dit qu'en 555/1160, à partir du Ğabal Ṭāriq (Gibraltar), «l'Autorité toute-puissante (c.-à-d. le calife 'Abd al-Mu'min) fit une sortie [...fa-harağa l-Amral-'aziz] contre les chrétiens» [T.m., p. 172, $1.1]$.

- Il est fait allusion aux šayh(s) des almohades «conseillers de cette Autorité toute-puissante (c.-à-d. du calife ${ }^{\mathrm{c}} \mathrm{Abd}$ al-Mu'min) [... an-nāsịhūn li-hād $\bar{a}-l-A m r$ al- $\left.{ }^{c} a z \overline{i z}\right] »[$ T.m., p. 177, 1.2].

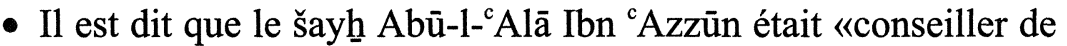
l'Autorité toute-puissante (c.-à-d. du calife ${ }^{\mathrm{c}} \mathrm{Abd}$ al-Mu'min) [... nasīh al-Amr al-c $\left.{ }^{c} a z \overline{i z}\right] »$. [T.m., p. 183, 1.10-11].

- Il est fait allusion au jour où la nouvelle du revers subi par les soldats du sayyid $\mathrm{Abū} \mathrm{Sa}^{\mathrm{c}} \overline{\mathrm{i}} \mathrm{d}$ dans la plaine de Grenade "parvint à sa Majesté l'Autorité toute-puissante (c.-à-d. au calife ${ }^{\mathrm{c}} \mathrm{Abd}$ al-Mu'min)... à Ribāt al-Fath (Rabat) [... wasala...ilä hadrati l-Amr al- ${ }^{c} a z \bar{z} z$.. bi-Ribät al-Fath]» [T.m., p. 193, 1.11].

- Il est signalé qu'après le siège de Murcia occupée par Ibn Mardanīš, les sayyid(s) Abū Hafș et $\mathrm{Abū} \mathrm{Sa}{ }^{c} \bar{i} d$ quittèrent les abords de la ville en 561/1165 mais y laissèrent un groupe d'almohades «qui la contrôlèrent pour le compte de l'Autorité toute-puissante (c.-à-d. du 
calife Abū Ya $\left.{ }^{c} \mathrm{qu} b\right)$ [... wa-ḍabața-hä li-l-Amr al- $\left.{ }^{c} a z \bar{z} z\right] »[$ T.m., p. 286, 1. 25].

- Il est dit qu'à l'occasion de la désignation du šayh Abū Yahyā Zakarīyā' comme wālī («gouverneur») de Séville en 561/1165, deux étendards, à lui destinés, avaient été noués «lors d'un conseil de l'Autorité toute-puissante (c.-à-d. du calife Abū Ya ${ }^{\mathrm{c}} q u \bar{b}$ ) [... fì mağlisi l-Amr al- $\left.{ }^{c} a z i \bar{z}\right] »$ [T.m., p. 294, 1.20].

- Il est fait allusion à une campagne menée en 562/1166 par le šayh Abū Sa ${ }^{c} \bar{d} d$ Yahllaf contre les Șanhāğa renégats «au nom de l'Autorité toute-puissante (c.-à-d. du calife Abū Ya $\mathrm{Ya}^{\mathrm{q}} \mathrm{qu} b$ ) [...bi-l-Amr al- $\left.{ }^{c} a z \bar{z} z\right] »$ [T.m. p. 328 - 1.12].

- Il est dit qu'en la même année 562/1166, le sayyid Abū Hafṣ, en campagne dans la montagne des Șanhāğa, obtint le ralliement de plusieurs tribus et «en informa l'Autorité toute-puisante (c.-à-d. le calife

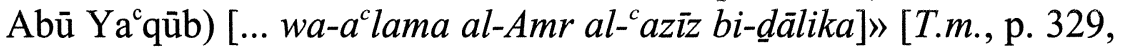
$1.5-6]$.

- Il est dit qu'en l'année 563/1167, pour demander renouvellement du serment d'allégeance, «l'Autorité toute-puissante (c.-à-d. le calife Abü $\left.Y^{c}{ }^{c} q u \bar{b}\right)$ fit parvenir une noble lettre au sayyid $A b \bar{u}$ Ibrāhīm Ismā̄īl à Séville [... nafada al-Amr al- ${ }^{c} a z \bar{z} z . .$. bi-kitāb karīm ilā as-sayyid...]» [T.m., p. 339, 1.4].

- Il est fait allusion au fait que le poète Abū 'Umar Ibn Harbūn savait, par ses vers, gagner l'intimité de «l'Autorité toute-puissante (c.-à-d. du calife Abū $\left.\mathrm{Ya}^{\mathrm{c}} \mathrm{qu} b\right)$ [... wa-taqarraba li-l-Amr al- $\left.{ }^{c} a z i \bar{z}\right] »$ [T.m., p. 367, 1.7].

- Il est dit qu'en 564/1168, l'Autorité toute-puissante (c.-à-d. le calife Abū Ya $\mathrm{a}^{\mathrm{c} q u ̄ b) ~ « l a v a ~ l e ~ v e ̂ t e m e n t ~ d e s ~ m o n t a g n e s ~ d u ~ G a r b ~ m a r o-~}$ cain de la souillure de l'hypocrisie [... wa-gasala al-Amr al- ${ }^{c} a z \bar{i} z .$. tiyäba-hā min danas an-nifäq]» [T.m., p. 375, 1.7].

- Il est dit que l'intervention de Ferdinand II El Baboso à Badajoz en 564/1168 fut un effet de la «bienveillance de Dieu et de son appui accordé à cette Autorité toute-puissante (c.à-d. au calife $\left.\mathrm{Abu} \mathrm{Ya}^{\mathrm{c}} q u \bar{b} \mathrm{~b}\right)$ [... bi-lutfi Alläh wa-ta'yidi-hi li-hādā l-Amr al- $\left.{ }^{c} a z \overline{i z}\right] »$ [T.m., p. 380, $1.8]$.

- Il est dit qu'après son ralliement aux almohades en 564/1168, Ibn Hamušk s'engagea «à se soumettre à l'Autorité toute-puissante et

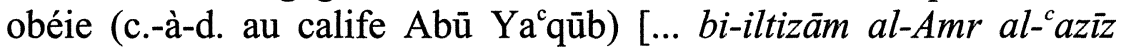
al-muțä $\bar{a}^{c}$ » [T.m., p. 389, 1.12]. 
- Il est dit qu'en cette même année 564/1168, l'Autorité toute-puissante (c.-à-d. le calife Abū Ya $\left.{ }^{\mathrm{c}} q u \bar{b}\right)$ «ordonna au hăfiz Abū Yahyā, gouverneur de Badajoz, de faire creuser un puits dans la qasaba de cette place [... wa-amara-hu al-Amr al- ${ }^{c} a z \bar{z}$ bi-hafr bi'r]» [T.m., p. 392, 1.9].

- Est évoquée, dans un titre, la réponse que firent les Arabes d'Ifrīqiya à l'Autorité toute-puissante (c.-à-d. au calife Abū Ya $\left.{ }^{c} q u ̄ b\right)$ lorsqu'il eut proclamé la mobilisation [Al-iğāba min al- ${ }^{c}$ Arab ilā al-Amr al- $\left.{ }^{c} a z i z z . ..\right] \gg[T . m .$, p. 417, 1.4].

- Il est dit que lors de la revue des Arabes à Marrakech en $566 / 1170$, on tint compte, «dans la façon de classer leurs tribus, de l'ordre d'antériorité de leur adhésion au tawhìd telle que déclarée à cette Autorité toute-puissante (c.-à-d. au calife Abū Ya ${ }^{\mathrm{c}} \mathrm{qu} \mathrm{u}$ ) [... ${ }^{c} a l \bar{a}$ tartīb tawhìdi-him awwalan fi qabā'ili-him as-säbiqa li-hädā l-Amr al- $\left.{ }^{c} a z \bar{\imath} z\right] »[$ T.m., p. 435, 1.5].

- Il est fait allusion à la grandiose parade militaite qui fut organisée à Marrakech en cette année 566/1170 «selon l'habitude de l'Autorité toute-puissante (c.-à-d. du calife $\left.\mathrm{Abū} \mathrm{Ya}{ }^{c} q u \bar{b}\right)\left[\ldots^{c} a l \bar{a}^{c} \bar{a} d a t i\right.$ l-Amr al- $\left.{ }^{c} a z i \bar{z}\right] »[$ T.m., p. 438, 1.20].

- Il est dit que certains partisans d'Ibn Mardanīš firent leur soumission en l'année 567/1171 à Cordoue et «se hâtèrent auprès de cette Autorité toute-puissante (c.-à-d. du calife $\mathrm{Abū} \mathrm{Ya}^{\mathrm{c}} q u \bar{b}$ ) [... wa-bidāri-him ilä häd̄a l-Amr al- ${ }^{c}$ aziż]» [T.m., p. 470, 1.6-7].

- Il est fait allusion à l'histoire de la mosquée d'Al-'Adabbas à Séville depuis son origine «jusqu'au jour où Dieu avait rassemblé l'Islam par l'intermédiaire de cette Autorité toute-puissante (c.à d. du

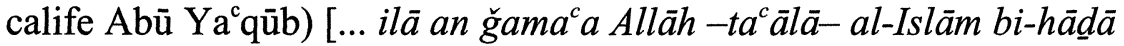
l-Amr al- ${ }^{c} a z \bar{z}$...]» [T.m., p. 475, 1.13 à p. 476, 1.1].

- Il est dit que lors du retour du calife Abū Ya ${ }^{c} q u ̄ b$ de son expédition de Huete en 567/1171, il passa par Játiva et que, dans cette ville, les $\breve{s} a y h(s)$ des almohades firent valoir à la population «le bénéfice qui se trouvait attaché pour eux au passage de cette Autorité toute-puissante [...wa-baššarū-hum bi-l-hayr al-muttașil min hāda l-Amr al- $\left.{ }^{c} a z i z\right] » ~[T . m .$, p. 513, 1.1-2].

- Il est dit que lors de ce même trajet-retour de l'expédition de Huete, en entrant dans Murcia, «l'Autorité toute-puissante (c.-à-d. le calife $\left.A b \bar{u} Y^{c} a^{c} q u ̄ b\right)$ avait incité Hilāl Ibn-Mardanīš à veiller à ce que les almohades fussent installés dans les demeures (de la ville) 
[... wa-qad kāna al-Amr al- ${ }^{c} a z \bar{z}$ nafada li-Hilāl b. Muhammad an yanzura fi inzāl al-muwaḥhidīn fi-d-diyār]» [T.m., p. 514, 1.5-6].

- al-Amr al- ${ }^{c} \overline{a l l}$ (al- ${ }^{c}$ alì): «la Haute-Autorité» ${ }^{12}$

- Il est dit que la Haute-Autorité (c.-à-d. le calife ${ }^{\mathrm{c}} \mathrm{Abd}$ al-Mu'min) témoigna sa bienveillance «aux ouvriers du chantier du Ğabal Tāriq (Gibraltar) lors de son passage sur place en l'année 555/1160 [...fa-hasana la-hum al-Amr al- $\left.{ }^{c} a ̈ l i\right]$ » [T.m., p. 171, 1.7-8].

- Il est dit qu'il y eut accord entre la Haute-Autorité (c.-à-d. le calife ${ }^{\circ} \mathrm{Abd}$ al-Mu'min) et les almohades «sur la désignation du sayyid $\mathrm{Abū} \mathrm{Ya}{ }^{c} q u \bar{b}$ comme héritier présomptif [... wa-ittifäq al-Amr al- ${ }^{c} a l \bar{\imath}$ wa-l-muwahhhidin]» [T.m., p. 207, 1.1].

- Il est mentionné, dans un titre, qu'en 563/1167, une baraka plénière fut dispensée par la Haute-Autorité (c.-à-d. par le calife Abū $\mathrm{Ya}^{\mathrm{c}} \mathrm{qū}$ ) [Al-baraka al- ${ }^{c} \bar{a} m m a$ an-näfid bi-hä al-Amr al- ${ }^{c} \overline{a l l}$...] [T.m., p. $353,1.1]$.

- Il est dit qu'en la même année 563/1167, Fernando Rodríguez, gendre d'Alphonse VII, fit séjour à Marrakech «sous régime de faveur de la part de la Haute-Autorité (c.-à-d. du calife $\mathrm{Abū} \mathrm{Ya}{ }^{c}$ qūb) [... taḥta ị̆sān min al-Amr al- $\left.{ }^{c} \bar{a} l \bar{i}\right] »$ [T.m., p. 369, 1.6-7].

- Il est dit qu'après le retour du dit Fernando en son pays, «la Haute-Autorité (c.-à-d. le calife $\mathrm{Abū} \mathrm{Ya}^{\mathrm{c}} \mathrm{qu} \mathrm{u}$ ) ordonna qu'on lui accordât subside ainsi qu'à ses frères et à ses compagnons [...wa-amara la-hu al-Amr al-'ālī... bi-muwāsāti-hi wa-muwāsät ihwwati-hi wa-aṣhäbi-hi]» [T.m., p. 370, 1.1]

- Il est dit que lorsqu'en 564/1168 Ibn Hamušk fit, par lettre, une proposition de ralliement aux almohades, «la Haute-Autorité (c.-à-d. le calife $A b \bar{u} Y a^{c} q u \bar{b}$ ) lui répondit en lui signifiant ses sentiments de rapprochement et de conciliation [... fa-ğāwaba-hu al-Amr al- ${ }^{c} a \bar{l} i . .$. bi-taqrībi-hi wa-istiğläbi-hi]» [T.m., p. 390, 1.2].

${ }^{12}$ C'est cette appellation qui est aussi attestée dans le titre de l'ouvrage non retrouvé d' al-Ašìīi (Abū ${ }^{c}$ Alī Hasan b. ${ }^{c} A b d$ Allāh b. Hasan Ibn al-Ašīin at-Tilimsānī) consacré à 'Abd al-Mu'min: Nazm al-la'älī fi futūh al-Amr al-'älì («Arrangement de perles dans le récit des victoires de la Haute-Autorité»). Ibn 'Id̄ārī, en donnant «le livre d'al-Ašīì̄» dans la liste de ses sources (Bayãn, I, p. 3, 1.3) n'indique pas son titre, lequel n'apparait pas non plus dans les pages (début de $B$. alm.) où al-Ašsīin est nommément cité (p. 17,1 . 10 ; p. $21,1.15$; p. 22 , 1.7 et 1.22 ; p. $26,1.19$; p. $28,1.14$; p. $47,1.3)$.. désalmohadisation oblige! Sur Hasan Ibn al-Ašīī et ses écrits, voir la Takmila d'Ibn al-Abbār, éd. Codera, F., Madrid, 1887-89, notice 66 . 
- Il est dit qu'en 565/1169, lors de son séjour à Cordoue, le sayyid Abū Ḥafṣ envoya son propre fils Abū Yahyā à Badajoz comme gouverneur de cette ville «de la part de la Haute-Autorité [... ${ }^{c}$ ani $l-A m r$ $\left.a l^{c} \bar{a} l i \bar{l}\right] \gg[$ T.m., p. 392, 1.5].

- al-Amr al-karim: «la Noble-Autorité»

- Il est fait allusion à un corps spécial de soldats andalous «inscrits au service privé de la Noble-Autorité (c.-à-d. du calife ${ }^{c} A b d$ al-Mu'min) [... al-marsümün fì zimām al-Amr al-karim]» [T.m., p. $183,1.11]$.

- Il est dit qu'ayant appris le revers essuyé par le sayyid Abū $\mathrm{Sa}^{\mathrm{c}} \mathrm{i} d$ dans la plaine de Grenade, «la Noble-Autorité (c.-à-d. le calife 'Abd al-Mu'min) sélectionna un énorme corps d'armée [... ihhtāra min-hum al-Amr al-karim 'askaran dahnm $\left.{ }^{a n}\right] »$ pour l'envoyer là-bas venger l'affront [T.m., p. 193, 1.14].

- Il est mentionné que lorsque le sayyid Abū Hafș alla, en 559/1163, rencontrer son frère $\mathrm{Abū} \mathrm{Sa}^{c} \overline{1} \mathrm{~d}$ au Ğabal TTāriq (Gibraltar), il emmena avec lui certaines hautes personnalités «de par la volonté de la Noble-Autorité (c.-à-d. d'A Abd al-Mu'min) [... bi-l-Amr al-karim]». [T.m., p. 251, 1.5].

- Il est dit qu'après les incidents qui avaient éclaté à Marrakech en 566/1170 parmi les Arabes rassemblés pour la prochaine expédition en al-Andalus, ceux-ci «se précipitèrent afin d'obtenir de la No-

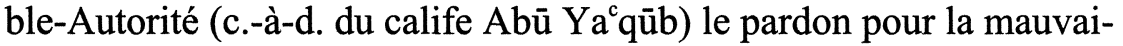
se conduite de leurs hommes [... țumma inna l-cArab tațaraḥu ${ }^{c}$ alä-l- ${ }^{c}$ afwi min al-Amr al-karim min qabịh mā ğanā-hu atb $\left.\bar{a}^{c} \bar{u}-h u m . ..\right] »[$ T.m., p. 434, 1.8-9]

- al-Amr al-mutāa : «l'Autorité-Obéie»

- Il est dit que l'inspection du Ğabal Ṭāriq (Gibraltar) en $555 / 1160$ se déroula «selon l'objectif assigné par l'Autorité-Obéie (c.-à-d. par le calife 'Abd al-Mu'min [... alā l-garad alladī nafad bi-hi al-Amr al-muța $\bar{a}^{c}$ )» [T.m., p. 171, 1.5].

- Il est dit qu'en l'année 556/1161, après la prise de Carmona par les almohades, le rebelle Ibn Abī Ğa far fut pris et retenu captif jusqu'à ce qu'il fût exécuté et "dressé au gibet par ordre de 1'Autorité-Obéie (c.-à-d. du calife 'Abd al-Mu'min [... ilā an nafada al-Amr al-muțac ... bi- șalbi-hi...]» [T.m., p. 185, 1.3-4]. 
- Il est dit qu'après la victoire des almohades à Grenade en $557 / 1162$, «ils remercièrent Dieu pour le secours qu'Il avait accordé à son Autorité-Obéie (c.-à-d. à 'Abd al-Mu'min) [...wa-šakarū Allāh

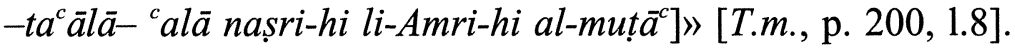

- al-Amr as-sa $a^{c} i d:$ «l'Heureuse-Autorité»

- Il est rappelé qu'en 557/1162 le dissident Ibn Hamušk contrôlait, dans la région de Séville, presque toutes les populations que Dieu avait précédemment fixées «dans l'obéissance à l'Heureuse-Autorité (c.-à-d. au calife ${ }^{\mathrm{C}} \mathrm{Abd}$ al-Mu'min) [... bi-ț-t $t \bar{a}^{c} a t$ 'alä l-Amr as-sacìd]» [T.m. p. 186, 1.10].

- (hādā) l-Amr al- ${ }^{c}$ azìim: «la Sublime-Autorité»

Non attestée — sauf inadvertance de ma part- dans le fragment retrouvé du Ta'rīh al-mann, cette appellation - avec ou sans häd $\bar{a}-$ apparaît plusieurs fois dans les textes de lettres califales.

- On la trouve, par exemple, dans la lettre adressée depuis Gafsa par le calife Abū Yūsuf $Y^{c}{ }^{c} q u \bar{b}$ al-Manșūr en 583/1179-1180 aux tālib(s) de Marrakech, dans laquelle louange est adressée à Dieu pour «les victoires remportées par cette Sublime-Autorité [... min futūḥ hāda $l$ l-Amr al- $\left.{ }^{c} a z i \bar{i}\right] \gg .{ }^{13}$

- hād $\bar{a}$ l-Amr al-gāalib: «cette Autorité-Victorieuse»

Non attestée — sauf inadvertance de ma part- dans le fragment retrouvé du Ta'rīh al-mann, cette appellation se trouve être utilisée à la fin du célèbre Fașl al-maqāl ${ }^{14}$ du quinquagénaire Ibn Rušd al-ḥafid (Averroès), lequel -il faut ici s'en souvenir - avait compo-

${ }^{13}$ Cf. Lévi-Provençal, E., Trente-sept lettres officielles almohades, Rabat, 1941, lettre 32, p. 199, 1.13. Autres exemples de cet al-amr al- ${ }^{\mathrm{c}}$ azimm: désignant ${ }^{\mathrm{C}} \mathrm{Abd}$ al-Mu'min: lettre 23, p. 136, 1.8; désignant $Y^{c}{ }^{c} q u \bar{b}$ al-Mansūr: lettre 29, p. 158, 1.9 et lettre 30, p. 180, 1.18. C'est ici le lieu de faire remarquer que dans ces lettres -éditées en 1941- nombreuses sont les utilisations des appellations que nous sommes en train de passer en revue; exemple de celle de $h a \bar{a} d \bar{a} l$-amr, désignant Abū Ya ${ }^{\mathrm{c}}$ qūb: lettre 25, p. 146, 1.15; exemples de celle d'al-amr al- ${ }^{c} a z \bar{z}$, désignant ${ }^{\circ} \mathrm{Abd}$ al-Mu'min: lettre 11, p. 38, 1.7; lettre 12,

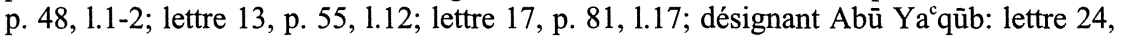
p. 139, 1.14; désignant $Y^{c}{ }^{c} q u \bar{b}$ al-Manșūr: lettre 28, p. 164, 1.9; lettre 29, p. 169, 1.9; exemples de celle de hādā l-amr al-karīm, désignant 'Abd al-Mu'min: lettre 18, p. 94, 1.6; lettre 19, p. 96, 1.11.

${ }_{14} \mathrm{Cf}$. 1. Ibn Rochd (Averroès), Traité décisif sur l'accord de la religion et de la philosophie, texte arabe et traduction française L. Gauthier, $3^{\text {ème }}$ éd., Paris, J. Vrin, 1983, p. 33. 2. Averroès, Le livre du discours décisif, introduction par A. de Libera, traduction inédite, notes et dossier par M. Geoffroy, Paris, GF-Flammarion, 1996, 170 - 71. 
sé, au temps de son enthousiaste jeunesse almohade, une Maqāla $f \grave{\imath}$ kayfiyyat duhüli-hi fi l-Amr al- ${ }^{c} a z i z$ («Explication de son entrée au service de l'Autorité toute-puissante»).

- Pour souligner le rôle qu'il reconnaît au souverain mu'minide almohade régnant dans l'apaisement des tensions précédemment existantes entre les acteurs de la vie intellectuelle musulmane, Averroès désigne ce souverain -à savoir le calife $A b \bar{u} \mathrm{Ya}^{\mathrm{c}} \mathrm{qu} \mathrm{u}$ - - selon l'usage de son temps par l'appellation de «cette Autorité-Victorieuse» et écrit: «Dieu a mis fin à beaucoup de ces maux, de ces ignorances et de ces cheminements menant à l'errance, grâce à l'action de cette Autorité-Victorieuse [... wa-qad rafaca Allāh kațiran min hädihi šššurūr wa-l-ğahalāt wa-l-masālik al-muḍillāt bi-hādā l-Amr al-ḡälib...]». ${ }^{15}$

\section{A la recherche de l'origine de cette utilisation mu'minide du mot $A m r$}

Ce passage en revue de nombreux contextes où est utilisée l'appellation d' "al-Amr», quelquefois seule mais le plus souvent enrichie d'une épithète glorifiante, tels qu'attestés dans le Ta'rīh al-mann et dans quelques autres textes d'époque, fait apparaître que l'emploi personnifié de cette appellation pour désigner le calife régnant fut général durant toute la durée du califat mu'minide. Mais il n'est pas moins évident pour les lecteurs et les utilisateurs des grandes sommes historiographiques postérieures aux almohades - le Bayān d'Ibn 'Id̄ārī, le Qirțās d'Ibn Abī Zar', le Kämil d'Ibn al-Ațīr, les ${ }^{c} I b a r$ d'Ibn Haldūn... etc., que dans les parties de ces ouvrages consacrées aux Almohades, la plupart des traces de cette utilisation mu'minide du mot $A m r$ ont disparu. Nous avons là un des effets les plus manifestes de ce que j'ai appelé ailleurs ${ }^{16}$ le procédé post-al-

15 L. Gauthier a traduit jadis ce bi-hāda la l-amr al-ġālib par «grâce au pouvoir établi» en expliquant (note 77) qu'il s'agissait de «la dynastie almohade». Plus récemment, M. Geoffroy et A. de Libera n'ont pas manqué d'attirer l'attention sur la dite expression (traduite par «ce pouvoir vainqueur» [trad.: $\S 72$ et note 147]; toutefois, en écrivant dans son Introduction (p. 54): «... grâce à ce qu' Ibn Rushd appelle ce pouvoir vainqueur...», A. de Libera semble attribuer à Averroès l'originalité de cette appellation alors que le philosophe ne faisait qu'utiliser, pour désigner son souverain et ami, une des formules qui avaient cours dans la société mu'minide de son temps.

16 Voir «Les talaba dans la société almohade», 331-33 
mohade de désalmohadisation; si le texte du Bayān almohade ${ }^{17}$ a conservé, prise à ses sources, une partie du «climat» mu'minide (ce qui lui valut d'être mis à l'index) ${ }^{18}$, les ouvrages qui le suivirent ont traité d'une dynastie almohade totalement nettoyée de ses spécificités. Aujourd'hui donc que la lecture de textes historiografiques almohades édités relativement récemment - comme le Ta'rịh al-mann - nous fait redécouvrir cette société almohade délibérément «oubliée», nous nous sentons dans l'obligation de relire des textes édités depuis plus longtemps (Mémoires d'al-Baydaq; Lettres almohades) dans l'espoir de les mieux comprendre! C'est ainsi que je suggère que soit trouvée l'origine de l'utilisation de l'appellation d' al-Amr pour désigner le calife mu'minide, dans l'interprétation d'une exclamation prêtée au mahdī Ibn Tūmart, exclamation bien connue mais comprise et expliquée autrement jusqu'à présent.

Nous sommes dans le récit de la bataille de la Buhayra ${ }^{19}$ en $524 / 1130$, lors de laquelle les Almohades furent sévèrement battus par les Almoravides sous les murs de Marrakech. Se mettant lui-même en scène, le témoin al-Baydaq ${ }^{20}$ écrit:

«Là, ${ }^{\mathrm{C}} \mathrm{Abd}$ al-Mu'min b. 'Alī, le [futur] Calife agréé d'Allāh, m'aborda et me dit: 'O Abū Bakr ${ }^{21}$, va en toute hâte mettre l'Imām au courant!' Je partis sans tarder rejoindre le Maître et lui rendis compte de l'évènement. ' $\mathrm{Abd}$ al-Mu'min est-il en vie?' me dit-il - 'Oui!' lui répondis-je. - 'Allāh, Maître des Mondes, soit loué! qad baqiya Amru-kum! A-t-il été blessé?' - 'Il a été atteint à la cuisse droite' - 'Il n'y a de force et de puissance qu'en Allāh, l'Elevé, le Magnifique! s'écria-t-il alors. $\boldsymbol{a l}-\boldsymbol{A} \boldsymbol{m} \boldsymbol{r}^{\boldsymbol{\prime}} \boldsymbol{b} \overline{\boldsymbol{a}} \boldsymbol{q}^{i{ }^{\prime}}$ !' Et il ajouta: 'Retourne vers lui et dis-lui:

17 Voir supra, note 10.

18 On a déjà parlé de la désalmohadisation modérée opérée par Ibn 'Id̄ārī (cf. supra: note 10). Ici, dans les pages (B. alm., éd. Casablanca, 1985, p. 63, 1.5 à p. 126, 1.6) où il a utilisé le $2^{\text {ème }}$ sifr (retrouvé) du T.m. et rencontré nos emplois d'al-Amr dans son sens personnifié de «l'Autorité» et désignant le calife, il n'en a retenu aucun. Dans une annexe mise à la suite de cet article, j'indiquerai ce qu'est devenu le texte 'id̄āien chaque fois que, dans ces pages, a été omis un $a l-A m r . .$. personnifié et je révélerai que, dans les pages antérieures (début de $B$. alm.), quelques emplois de cet $a l-A m r$ désignant le calife ont été retenus.

19 Cette bataille de la Buhayra fut assimilée par les almohades à la bataille d'Uhud. Cf. Kably, M., Société, Pouvoir et Religion au Maroc, Paris, 1986, p. 60, note 1.

${ }^{20}$ Les Mémoires d'al-Baydaq. Éditions: 1. Lévi-Provençal, E., dans Documents inédits d'histoire almohade, Paris, Geuthner, 1928, texte arabe, p. 79; trad. française: pp. 128-9. 2. Ahbār al-mahdī Ibn Tūmart, Rabat, Dār al-Manșūr, 1971. 3. 'Abd-al-Hamīd Hāğiyāt, Kitāb ah̆bār al-mahdī IbnTūmart. Alger, S.N.E.D, 1975.

${ }_{21}$ Abū Bakr b. 'Alī as-Ṣanhāğĩ: c'est al-Bayḍaq. 
$\boldsymbol{a l}-\boldsymbol{A} \boldsymbol{m} \boldsymbol{r}^{\mu} \boldsymbol{b} \overline{\boldsymbol{a}} \boldsymbol{q}^{i n}$ ! Ne vous affolez pas!' Je revins auprès de 'Abd al-Mu'min et lui rapportai les paroles du Maître. Il se réjouit et nous nous réjouîmes».

La traduction de ces lignes est de Lévi-Provençal (voir note 20) ${ }^{22}$, à cette réserve près que $\mathrm{j}$ 'y ai intentionnellement maitenu en arabe l'exclamation martelée trois fois par Ibn Tūmart avec chaque fois le mot Amr: «qad baqiya Amru-kum» - «al-Amr bā $\left.q^{i n}\right\rangle-\left\langle a l-A m r b \bar{a} q^{i n}\right\rangle$, dont je propose de réexaminer la signification.

Lévi-Provençal a traduit par «Rien n'est perdu pour vous!» «Rien n'est perdu!» - «Rien n'est perdu! Ne vous affolez pas!», en prenant, de toute évidence, le mot $A m r$ (plur. umür) dans son sens de «chose», d' «état de chose», de «situation», et en donnant à qad baqiya Amru-kum la signification de «votre situation reste intacte!» et à $a l-A m r b \bar{a} q^{\text {in }}$ celle de «la situation reste intacte!», d'où le «rien n'est perdu!» adopté comme traduction ${ }^{23}$.

Je pense qu'il faut ici donner à $A m r$ (plur. awämir) son sens d' «Autorité». Alors, la triple exclamation, sur les lèvres d'Ibn Tūmart à qui l'on apprenait que son lieutenant ' $\mathrm{Abd}$ al-Mu'min n'était pas mort, prend toute sa signification et toute son importance:

«Allāh, Maître des mondes soit loué! (Celui qui est) votre Autorité est resté vivant! ... L'Autorité reste (vivante)! ... l'Autorité reste (vivante)!»

Comprise ainsi, il me paraît raisonnable de penser que cette exclamation, martelée trois fois - qu'elle soit historiquement véridique ou qu'elle ait été forgée par ${ }^{\mathrm{C}} \mathrm{Abd}$ al-Mu'min et ses partisans- puisse être à l'origine de l'habitude prise par les Mu'minides de donner à ' $\mathrm{Abd}$ al-Mu'min et à chacun de ses successeurs, à côté des appellations de

22 Dans cette traduction, on peut s'étonner qu'Ibn Tūmart soit une fois appelé l'Imām et deux fois «le Maître» alors que dans le texte arabe, un seul mot sert à le désigner: $a l-M a^{c}$ șüm que je comprends comme «l'Immunisé», préférant éviter les habituelles traductions de «l'Infaillible» ou «l'Impeccable».

${ }^{23}$ Il est probable que je ne suis pas le premier à remettre en cause cette traduction par Lévi-Provençal de l'exclamation d'Ibn Tūmart; d'autres lecteurs - arabophones ou arabisants - ont dû sourciller avant moi! Par contre, nombreux sont ceux qui ont repris le récit de la bataille de la Buhayra tel que traduit jadis par le grand professeur; voir Bourouiba, R., Ibn Tümart, Alger, S.N.E.D., 1974, p. 72; et ' $A b d$ al-Mu'min flambeau des Almohades, Alger, S.N.E.D., 1974, p. 16. Tout récemment, C. Durix, dans son excellent petit livre sur ${ }^{~} A b d$ as-Salām Ibn Mašǐšs, saint-homme d'époque almohade, Le Maroc et le Saint (Paris, Le Cerf, 2000) écrit à propos de la bataille de la Buhayra (pp.72-3): «Une sortie audacieuse des Almoravides leur permet d'écraser l'armée almohade. 'Abd al-Mu'min a la vie sauve et Ibn Tūmart déclare "Ne nous affolons pas, rien n'est perdu!». 
«Calife» (halïfa), de «Commandeur des croyants» (Amir al-Mu'mininn) ou de «sayyid suprême», celle de «l'Autorité». Peu de temps avant sa mort, Ibn Tümart avait ainsi transmis le relais du Amr à son lieutenant, survivant de la bataille de la Buhayra: 'Abd al-Mu'min. On n'est donc pas étonné de constater que l'écho d'une exclamation du Mahdi politiquement si riche de sens se soit répercuté dans les écrits des historiographes almohades et, ensuite, dans ceux de certains historiens post-almohades ${ }^{24}$. A ces derniers, plus ou moins désalmohadisateurs, 1' hétérodoxie de la formule rapportée par le témoin al-Baydaq posa problème: peu la transmirent telle quelle avec les deux mots d'al-amr et de baqiya $\left(b \bar{a} q^{i n}\right)$; certains l'édulcorèrent et d'autres la passèrent sous silence. Considérée par la suite comme taboue par les lecteurs marocains et mal comprise de nos jours par les arabisants maghribistes, elle mérite qu'on lui retrouve son sens historique.

\section{Ont transmis l'exclamation avec les deux mots exprimés de amr et de baqiya (ou $b \bar{a} q^{i n}$ ):}

— Ibn Ṣāḥib aṣ-Ṣalāt très vraisemblablement (écrivant sous le règne du deuxième calife, $\mathrm{Abu} \mathrm{Ya}^{\mathrm{c}}$ qūb Yũsuf), dans le $1^{\mathrm{er}}$ sifr (non retrouvé) du Ta'rīh al-mann, si nous en croyons l'auteur des al-Hulal al-mawšiyya ${ }^{25}$ qui le cite: «Selon ce qu'a mentionné Ibn Șāḥib aș-Ṣalāt, Ibn-Tūmart s'exclama: 'Du moment qu' 'Abd al-Mu'min est en vie, c'est l'Autorité qui est restée vivante!' [... qāla: 'mundِu 'āša 'Abd al-Mu'min baqiya l-Amr'...]».

- Abū Muḥammad Ḥasan Ibn al-Qațtān [Ibn al-Qațāān fils] (écrivant sous le règne du onzième calife, al-Murtaḍā), dans son Nazm al-ğumān, qui l'a donnée sous deux formes: une première fois ${ }^{26}$ en faisant dire à Ibn Tūmart apprenant qu' 'Abd al-Mu'min est vivant: «C'est comme si personne n'était mort, et la divine bénédiction (se trouve manifestée) dans le fait qu'il soit resté en vie [...ka-anna-hu lam yamut aḥad wa-l-baraka fí baqā' $i$-hi...]» et une seconde ${ }^{27}$ en le

${ }^{24}$ Lévi-Provençal avait bien constaté que l'exclamation d'Ibn Tūmart rapportée par al-Baydaq se trouvait répercutée dans de nombreux ouvrages postérieurs. Voir, dans sa traduction: p. 128 , note 2 .

${ }^{25}$ Voir ci-dessous.

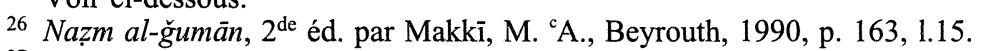

27 Op. cit., p. 171, 1.2-5. 
faisant s'écrier: «Alors, l'Autorité (se trouve assurée) de rester (vivante) jusqu'à l'échéance de l'heure! [... fa-l-Amr bāaqn ilā qiyām $\left.a s-s \tilde{a}^{c} a\right]$ » ${ }^{28}$, pour faire ensuite ce commentaire: "cette parole et d'autres semblables prononcées par l'Imām Mahdī —que Dieu le Très-Haut l'ait en agrément! - ont été confirmées par la réalité puisqu'une lignée de califes s'est mise en place après lui ${ }^{29}$ - que Dieu le Très-Haut les ait tous en agrément!- (qui perdurera) jusqu'à l'échéance de l'heure grâce à l'omnipotence de Dieu le Très-Haut».

- Ibn 'Id̄ārī al-Marrākušì, auteur de l'al-Bayān al-muǵrib, l'un des premiers utilisateurs post-almohades (début des Mérinides) des écrits almohades (et en particulier du Ta'rịh al-mann et du Nazm al-ğumān) qui, évoquant la défaite de la Buhayra ${ }^{30}$ a terminé son récit par ces mots: «'Abd al-Mu'min se dirigea alors vers Tìnmal en compagnie de cinquante hommes»; il trouva le Mahdi qui leur dit: «l'Autorité est restée vivante! [... baqiya $l-A m r] »{ }^{31}$. Ibn 'Idārī a été ici égal à lui-même; sa préoccupation de désalmohadiser ne l'a pas em-

${ }^{28}$ En 1922 -donc avant le «rien n'est perdu!» de la traduction d'al-Baydaq en 1928Lévi-Provençal avait traduit: «Alors notre fortune se maintiendra jusqu'à l'arrivée de l'heure (du jugement dernier)». Cf. "Six fragments inédits d'une chronique anonyme du début des Almohades», Mélanges René Basset, Paris, 1925, 335-93; voir fragment VI.

${ }^{29}$ Ibn al-Qatțān écrivait durant le règne du calife al-Murtaḍā, un siècle après la mort d'Ibn Tümart.

${ }^{30}$ Il y a dans le Bayān almoravide (B. IV, édition d' 'Abbās, I., Beyrouth, 1967) deux évocations complémentaires de la défaite almohade de la Buhayra: la première (p. 75, 1.19 à p. 76, 1.13), inspirée d'Ibn al-Qattān (cité) (voir Nazm al-ğumān, pp. 160-3) logée à l'année $521 \mathrm{~h}$. dans la chronique almoravide, résume les affrontements entre Almohades (Tīnmal) et Almoravides (Marrakech) qui se déroulèrent à partir de $521 \mathrm{~h}$. et dont le dernier acte (seule défaite après quarante victoires) fut la défaite almohade de la Buhayra (sans que l'année $524 \mathrm{~h}$. soit ici indiquée); la seconde (p. 83,1.15-20) prise à Ibn Ḥamāduh as-Sabtī (cité), logée dans la chronique de l'année $524 \mathrm{~h}$., rappelle le long siège de Marrakech qui se termina par «une bataille (le nom d'al-Buhayra n'est pas indiqué) qui eut lieu entre eux et lors de laquelle moururent, parmi les Almohades, tous ceux dont Dieu avait fixé le jour!».

${ }^{31}$ Cf. B. IV, p. 76, 1.12-13. Huici Miranda et après lui Maḥmūd 'Alī Makkī (Nazm, p. 161, notes 1 et 3 ) ont attiré l'attention sur les divergences existantes entre la relation d' Ibn al-Qațāan et le récit d'al-Baydaq. Je remarque à mon tour que sous la plume d'Ibn 'Id̄ārī, utilisateur du Nazm, la contraction du texte de sa source a pour effet de faire arriver ${ }^{\mathrm{C}} \mathrm{Abd}$ al-Mu'min à Tìnmal «avec» $\left(m a^{c} a\right)$ les «cinquante hommes», alors que ceux-ci, pour Ibn al-Qatțān $(\mathrm{Nazm}$, ibid., p. 163, 1.2) avaient reçu l'ordre de gagner Tīnmal les premiers, au plus vite. Nous notons sans étonnement qu'A. Huici Miranda, dans le sillage de Lévi-Provençal, a traduit: «... quien le dijo: ha quedado la cosa!». Cf. Ibn 'Id̄ārī, Al-Bayān al-mugrib, Nuevos fragmentos almorávides y almohades, traducidos y anotados por A. Huici Miranda, Valencia, 1963 (Textos medievales, 8), p. 176. 
porté sur sa volonté de témoigner d'un moment original de l'histoire du Magrib: il a transmis littéralement l'exclamation d'Ibn Tümart.

- L'auteur (qui reste inconnu) des al-Hulal al-mawšiyya, grenadin du $8^{\text {ème }}$ (XIV'̀me) siècle ${ }^{32}$, citant explicitement Ibn Șāḥib aș-Șalāt ${ }^{33}$ : «... selon ce qu'ont mentionné Ibn Șāhib aș-Șalāt et d'autres auteurs, (le Mahdi) s'écria: 'du moment qu' 'Abd al-Mu'min est en vie, c'est l'Autorité qui est restée vivante!' [... qāla: "mundu 'āša 'Abd al-Mu'min baqiya l-Amr" dakara d̄ālika Ibn Șāhib aș-Ṣalāt wa-g்ayru-hu]». ${ }^{34}$

\section{Ont transmis l'exclamation en l'édulcorant mais en gardant trace d'un des deux mots: amr ou baqiya:}

- Ibn al-Atīir, contemporain syrien des Almohades, dans son Kãmil, qui a écrit: "C'est comme si personne n'était mort! (Le détenteur de) l'Autorité est resté debout! C'est lui qui va conquérir le pays [... mā māta aḥad! al-Amr qā'im wa-huwa allad̄i yaftạ̣u l-bilād]». ${ }^{35}$

- Ibn Haldūn, dans les 'Ibar, qui a écrit ${ }^{36}$ : il s'exclama: «puisque est resté vivant 'Abd al-Mu'min, c'est comme si personne n'avait péri! [... fa-qāla: '(id̄ā) mā baqiya 'Abd al-Mu'min fa-lam yahlik $a h a d$ ']» ${ }^{37}$. Ainsi banalisée, l'exclamation du Mahdī ne pouvait irriter ni les Mérinides ni les Hafsides! Comme l'on sait que les textes d'Ibn Haldūn concernant l'histoire du Magrib furent connus, en Europe, dès le milieu du XIX ème siècle (les 4 vol. de traduction de De Slane, Histoire des Berbères, sont de 1852-1856), il n'est pas interdit de penser que la lecture, ici, de l'exclamation telle que donnée par le génial his-

32 Sur cet auteur et les circonstances de la composition de son ouvrage, voir «Les talaba dans la société almohade», pp. 341-42 et notes 38 et 39 .

${ }^{33}$ Kitäb al-hulal al-mawšiyya fí dikr al-ah̆bār al-marrākušiyya, éd. par Suhayl Zakkār et 'Abd al-Qādir Zamāma, Casablanca, 1979, p. 116, 1.10-11.

${ }^{34}$ Notons ici aussi qu'assez tôt traduite par A. Huici Miranda, l'exclamation du Mahdi devenait sous sa plume: «Si vive 'Abd al-Mu'min, nuestra causa se ha salvado!», al-Hulal al-mawšiyya, trad. Tetuan, 1951, p. 139.

${ }^{35}$ Al-Kāmil, éd. an-Nağğãr, 'A. al-W., Le Caire, 1356/1930, VIII, p. 198, 1. 25. Dans ses Annales du Maghreb et de l'Espagne (Alger, 1898) E. Fagnan a traduit ainsi (p. 537): «C'est, dit-il, comme si personne n'était mort; rien n'est perdu, et c'est à lui qu'est réservé la conquête!». Ce «rien n'est perdu» de Fagnan, en traduction du al-amr qã 'im, a donc précédé d'une trentaine d'années celui de Lévi-Provençal!

36 'Ibar, éd. Beyrouth, VI, 260, 1.11.

37 C'est moi qui restitue le $i \underline{d} \bar{a}$. De Slane a jadis traduit: «Puisqu' Abd-el-Moumen est encore en vie nous n'avons rien perdu». Hist. des Berbères, I, 254. 
torien influença les lecteurs et traducteurs des textes almohades retrouvés plus tard (Mémoires d'Al-Baydaq et autres....)!

\section{Avait (le premier?) neutralisé l'exclamation en n'y retenant ni amr ni baqiya:}

— ${ }^{c} A b d$ al-Wāḥid al-Marrākušĩ dans son $M u^{c}$ ğib, rédigé au temps même des Mu'minides almohades mais au Mašriq, qui avait écrit: Il leur demanda: «'Abd al-Mu'min ne s'en est-il pas sorti sain et sauf?» - «Si!» - «Alors, (c'est comme si) personne n'avait été perdu!» [... qāla: a laysa qad nağ $\bar{a}^{c}$ Abd al-Mu'min? - qālü: nac am - qāla: lam yufqad ahad] ${ }^{38}$. Très fier de faire connaître le Magirib almohade aux mašriquins, l'auteur du $M u^{c}$ ğib n'était pas allé jusqu'à leur communiquer, dans sa littéralité hétérodoxe, l'exclamation d'Ibn Tūmart ${ }^{39 !}$

\section{Ont totalement passé l'exclamation sous silence pour d'évidentes raisons politiques:}

- tant les auteurs «dynastiques» mérinides, contemporains d'Ibn 'Iḍārī ou postérieurs à lui: Ibn Abī Zarc, auteur du Qirțās ${ }^{40}$, l'auteur de l'ad-Dahira as-saniyya ${ }^{41}$, al-Malzūzī dans son Nazm as-sulūk ${ }^{42}$, Ibn al-Ahmar dans son Rawdat an-nisrin 43 ... et d'autres.

- que les auteurs d'obédience hafside tunisienne et donc en rupture avec l'«Autorité» mu'minide marrākušienne, antérieurs à Ibn Haldūn: az-Zarkašì dans son Ta'rịh ad-dawlatayn ${ }^{44}$, Ibn Qunfud dans l'al-Fārisiyya ${ }^{45}$... et d'autres.

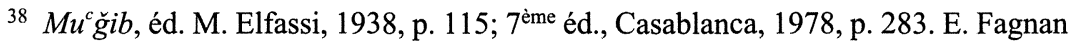
(Histoire des Almohades, Paris, 1893, p. 167) avait traduit: «Alors, c'est comme si personne n'était mort!».

39 Sur les circonstances de l'exil au Mašriq d d $^{\text {c }} \mathrm{Abd}$ al-Wāḥid al-Marrākušǐ, cf. «Les talaba dans la société almohade», p. 335 et note 15 .

40 Éd. Tornberg, C. J., Upsala, 1843; nombreuses traductions.

41 Éd. Rabat, Dār al-Manșūr li-ț-ṭibāa a wa-l-wirāqa, 1972.

42 Éd. Ben Manșūr, ${ }^{\mathrm{C}} \mathrm{A}$. al-W., Rabat, 1963.

${ }^{43}$ Histoire des Beni-Merin, éd. et trad. annotée par Bouali, G., et Marçais, G., Paris, 1917. Ed. Ben Manșūr, 'A. al-W., Rabat, 1962.

${ }^{44}$ Ta'rīh ad-dawlatayn al-muwahhidiyya wa-l-hafsiyya, éd. Tunis, 1289 h. Trad. Fagnan, Chronique des Almohades et des Haf̧̧ides attribuée à Zerkechi, Constantine, 1895.

${ }_{45}$ Al-Färisiyya fi mabädi' ad-dawla al-hafșiyya, ms. Escurial, éd. et trad. partielle par Cherbonneau dans Journal Asiatique, 1848-1852. 
Par la suite, le temps passant et la désalmohadisation faisant son œuvre, l'exclamation d'Ibn Tūmart au lendemain de la défaite de la Buhayra ne fut plus rapportée d'après le texte d'al-Baydaq; on 1' «oublia». Il reste que pour nous, lecteurs des quelques textes almohades qui nous sont parvenus et qui l'ont rapportée -le Ta'rīh al-mannn, le Nazm al-ğumān-, lecteurs du Bayān et des al-Hulal al-mawšiyya, nous avons compris que si ce cri du Mahdī a été par eux répercuté dans sa littéralité, c'est qu'il voulait dire autre chose qu'un «rien n'est perdu» si pathétique soit-il! Ce cri était le mot fort de celui qui bientôt allait mourir et qui donnait «Autorité» à 'Abd al-Mu'min: «(Celui qui est) votre Autorité est resté vivant!»

$\mathrm{Au}$ XIXème siècle, l'historien marocain an-Nāsirī as-Salawī (m. en 1897), dans ses pages sur les Almohades ${ }^{46}$, ignore aussi bien le texte d'al-Baydaq que les pages d'Ibn Șāhib as-Salāt. C'est chez Ibn Haldūn qu'il va chercher le récit de la Buhayra et y recopie mot à mot le dialogue entre les survivants et Ibn Tümart 47 avec l'exclamation finale de celui-ci donnée ci-devant: «(id̄a $) m \bar{a}$ baqiya ${ }^{\mathrm{c}} \mathrm{Abd}$ al-Mu'min fa-lam yahlik ahad». Nous constatons aussi que, toujours à la suite d'Ibn Haldūn, il n'hésite pas à compromettre les Almohades, possesseurs d'un exemplaire du Kitāb al-Ğafr, le fameux ouvrage d' ésoté-

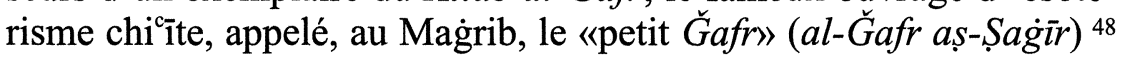
«dans lequel Ibn Tūmart avait lu que son Autorité (Amru-hu) n'atteindrait sa plénitude que dans les mains d'un homme portant tel nom et ayant telle allure, à savoir ${ }^{\mathrm{c}} \mathrm{Abd}$ al-Mu'min b. ${ }^{\mathrm{c}} \mathrm{Ali}[\mathrm{i}$...wa-kāna fì-hi anna Amra-hu lā yutammu illā calā yadi rağulin ismu-hu kad̄a wa-hilyatu-hu kadā wa-huwa 'Abd al-Mu'min b. ' $A l i \overline{]}] "{ }^{49}$.

Mais pour les lecteurs des textes almohades mu'minides, cette prédiction qu'aurait trouvée Ibn Tūmart dans le «petit Ğafr» n'est que l'une des nombreuses «traditions» colportées avec ferveur qui ont pour point commun d'affirmer qu' $\mathrm{Abd}$ al-Mu'min reçut du Mahdi, détenteur $\mathrm{du}$ charisme prophétique, la lieutenance $\mathrm{du} A m r$ «l'Autorité», c'est-à-dire se trouva légitimé à instaurer un Califat

${ }^{46}$ Kitāb al-Istiqșä', Casablanca, 1954, II, 76-256.

47 Id., p. 87, 1.20-23

${ }^{48}$ Cf. la Muqaddima d'Tbn Haldūn, éd. Beyrouth, I, 201, 1.13-16. Trad. franç. Monteil, V., Beyrouth, 1968, II, 697. Voir en $E I^{2}$, II, l'article «Djafr» de T. Fahd.

${ }^{49}$ Kitäb al-Istiqsă', II, p. 97, 1.21 à 23. 
dynastique autoritaire. Voici quelques-uns de ces «dires» qui confortent le qad baqiya Amru-kum dans son vrai sens:

Dans les «Mémoires» d'al-Baydaq, on lit qu'un devin de Tlemcen avait dit à la mère d ${ }^{\prime c} \mathrm{Abd}$ al-Mu'min: «Rien n'empêchera que cette femme ait un enfant dont l'Autorité enveloppera l'est, l'ouest, le sud et le nord! [Lä budda li-hädihi l-mra'a min mawlüd yakün Amru-hu ya'hudu l-mašriq wa-l-magrib wa-l-qibla wa-l-ğawf]» ${ }^{50}$. On y apprend aussi qu'après qu'c $\mathrm{Abd}$ al-Mu'min eut rencontré Ibn Tūmart à Mallāla, une nuit, en présence du seul al-Bayḍaq qui tenait la lampe, Ibn Tūmart déclara: «L'Autorité (al-Amr) qui aura en elle la vie de la religion ne s'édifiera que par ${ }^{\mathrm{C}} \mathrm{Abd}$ al-Mu'min, lampe des Almohades! [Lā yaqüm al-Amr allad̄ī fì-hi hayāt ad-dīn illā bi- ${ }^{c} A b d$ al-Mu'min $b .{ }^{c} A l i ̄$ siräğ al-muwahhhidīn]» ${ }^{51}$, et que, le matin venu, ayant réuni ses jeunes élèves il s'exclama devant eux: «Dieu est divinité unique; le Messager est vérité; le Mahdi est vérité; le calife est vérité. Lisez donc le hadịt d'Abū Dāwūd ${ }^{52}$ et vous (re)connaîtrez

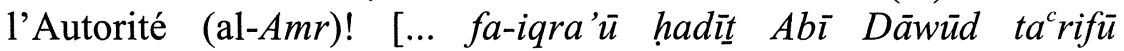
$a l-A m r] \gg{ }^{53}$.

Dans le $M u^{c} \check{g} i b$ d'Abd al-Wāḥid al-Marrākušì -lequel, avons-nous vu, a préféré ne pas révéler le al- $A m r b a \bar{q} q^{\text {in }}$ tūmartien à ses lecteurs mašriquins- plusieurs autres paroles d'Ibn Tümart sont néanmoins citées qui exaltent la notion de l' «Autorité» (al-Amr) dont les Almohades, par le truchement d ${ }^{\prime c} A b d$ al-Mu'min reçurent le dépôt. "L' 'Autorité' (al-Amr), leur répétait-il, ne cessera d'être entre vos mains jusqu'à l'échéance de l'heure! [... wa-lā yazäl al-Amr fí-kum ilā

50 Al-Bayḍaq, éd. Lévi-Provençal, 1928, texte arabe p. 54, 1.10.; éd. Alger, 1974, p. 39, 1.11-12. Ici dans sa traduction (p. 83), Lévi-Provençal a bien pris le mot amr dans son sens d'«autorité»: "Cette femme aura certainement un enfant dont l'autorité s'étendra à l'est, à l'ouest, au sud et au nord!»

51 Id., éd. Lévi-Provençal, texte arabe p. 56, 1.9-10; éd. Alger, p. 41, 1.14-15. Ici, Lévi-Provençal prend le mot $a m r$ dans le sens de «situation», d' "état de chose», de «mission» et traduit (p. 87): «La mission sur quoi repose la vie de la religion ne triomphera que par ${ }^{\mathrm{c}} \mathrm{Abd}$ al-Mu'min b. ${ }^{\mathrm{C}} \mathrm{Ali}$, flambeau des Almohades»; traduction reprise en anglais par Roger Le Tourneau: «The mission on which depends the life of the religion will succeed only by 'Abd al-Mu'min, the torch of the Almohades» (The Almohad Movement in North Africa in the Twelfth and Thirteenth Centuries, Princeton, 1969, p. 18).

52 Abū Dāwūd as-Siğistānī (m. en 275/889) auteur du Kitāb as-sunan.

53 Éd. Alger, p. 42, 1.4-6. Trad. Lévi-Provençal, p. 87: «Lisez le hadīị d'Abū Dāwūd, vous saurez ce qu'il en est!» reprise par R. Le Tourneau, p. 18: «Read Abû Dawud's hadith. You will know what he says about that». 
qiyām as-sāa $a] \gg{ }^{54}$. Il aimait à discourir, est-il aussi noté, sur la «la pérennisation de cette Autorité [... fi tahlìd hāâa l-Amr]» ${ }^{55}$, lançant peut-être ainsi l'usage de l'expression intensive (avec $h \bar{a} \underline{d} \bar{a}$ ) dont on a relevé l'usage dynastique chez les mu'minides. Concernant directement la façon de désigner ${ }^{\mathrm{c}} \mathrm{Abd}$ al-Mu'min, on peut lire ceci: «Il donna autorité sur l'armée à 'Abd al-Mu'min b. 'Alī et déclara: 'Vous êtes les croyants (mu'minin), lui est votre $A m \bar{i} r$ ', et à partir de ce jour-là, 'Abd al-Mu'min mérita le nom d'Autorité des croyants (Amr(at) al-mu'minin) [Wa-ammara 'alā l-ğayš 'Abd al-Mu'min b. 'Alī wa-qāla: antumu l-mu'minūn wa-hādā Amìru-kum; fa-istahaqqa 'Abd al-Mu'min min yawmi'idin ism amrat al-mu'minin]» ${ }^{56}$; on a là, de toute évidence, la légitimation tūmartienne de l'appellation donnée ensuite à 'Abd al-Mu'min d'Amir al-Mu'minin (Commandeur-descroyants) mais aussi de celle, plus spécifique, d'«al-Amr» («l'Autorité»). Cette légitimation est confirmée dans un texte donné pour le testament d'Ibn Tūmart où l'on peut lire: «Nous avons fait de lui (d'A Abd al-Mu'min) un Amìr à votre tête!... L'Autorité, c'est l'Autorité de Dieu! Il en investit qui il veut parmi ses serviteurs! [... wa-ğacalnā-hu Amīran calay-kum !... wa-l-Amr Amr Allāh yuqallidu-hu man š̃ $\tilde{a}^{c} a$ min $\left.{ }^{c} i b \bar{a} d i-h i\right] »{ }^{57}$.

Dans le Kämil du mašriquin Ibn al-Atìr -renseigné par des expatriés de l'Occident almohade du genre précisément d'A Abd al-Wāhid al-Marrākušs̄ ${ }^{58}$ - où, avons-nous vu, le al-Amr bā $q^{\text {in }}$ est devenu al-Amr $q \bar{a}^{\prime} i m$, nous lisons, tout de suite après, qu'Ibn Tümart «recommanda à ses compagnons de suivre 'Abd al-Mu'min, de le mettre à leur tête, de

${ }^{54} \mathrm{Cf} . M u^{c} g \check{i} i b, 7^{\text {ème }}$ éd., Casablanca, 1978, p. 276, 1.9. C'est l'écho de cette même parole que l'on a trouvé chez Ibn al-Qatțān (cf. ci-dessus et note 28). Fagnan, E. (Hist. des Almohades, Paris, 1893, p. 163) a traduit: «... c'est à vous que restera le commandement jusqu'à l'arrivée de l'heure suprême».

${ }_{55} M u^{c}$ ğib, p. 277, 1.2-3. E. Fagnan a traduit (p. 164): «... discours... d'Ibn Toūmart... qui sont relatifs à la perpétuité de l'autorité des Almohades...».

${ }^{56} M u^{c}$ ğib, p. 282, 1.7-9. E. Fagnan (ibid.) a traduit (p. 166): «Il leur donna pour chef 'Abd el-Mou'min b. 'Alī, en disant qu'ils étaient les croyants [mou'minoun], et que c'était là leur émir; aussi ' $\mathrm{Abd}$ el-Mou'min prit-il le titre d'Emir des croyants».

57 Ibid., p. 287, 1.7 et 1.14-15. E. Fagnan (ibid., p. 170-171) a traduit: «Nous avons choisi l'un d'entre vous pour en faire votre chef...... que le Seigneur suprême investisse qui Il voudra d'entre ses serviteurs!».

58 Parmi ces "occidentaux» expatriés à cette époque au Mašriq, à côté

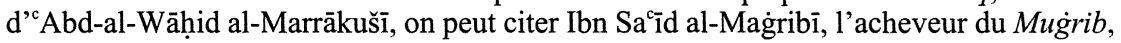
et le qāḍī sévillan Abū Marwān al-Bāğî, informateur d' Ibn Abī Ușaybica dans sa fameuse Histoire des médecins. 
lui remettre l'autorité (... taslìm al-amr ilay-hi), de se laisser conduire par lui et de lui donner le laqab d'Amir-al-Mu'minin', et qu'ensuite il mourut» 59 .

Que les recommandations testamentaires de leur Mahdi aient justifié chez les Mu'minides almohades l'utilisation de l'appellation «califale» d'Amīr al Mu'minīn, Ibn al-Atīir en fut informé et en témoigna, mais sut-il, chez lui en Syrie, qu'ils avaient été, pour désigner leurs califes, jusqu'à utiliser les appellations personnifiées d'al-Amr, de hādā l-Amr, d'al-Amr al- ${ }^{c} a z i \bar{z}$, d'al-Amr al-'älì, d'al-Amr al-karīm, d' al-Amr al-mutāa d'al-Amr as-sa'id, d'al-Amr al-'azim, d'al-Amr

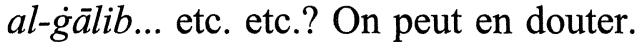

Aujourd'hui par contre, pour quiconque prend connaissance dans les textes mu'minides retrouvés ${ }^{60}$ des allusions faites à cette "autorité» (amr) dont 'Abd al-Mu'min aurait reçu le dépôt par le truchement de déclarations successives prononcées par le $M a h d \bar{l}$, il apparaît avec évidence que c'est le souvenir vrai ou la dévote élaboration écrite de ces supposées déclarations qui engendrèrent chez les mu'minides l'audacieuse et officielle habitude d'utiliser l'appellation courante de «l'Autorité» (al-Amr) pour désigner d'abord 'Abd al-Mu'min, puis chacun de ses successeurs. Mais alors, il ne semble guère possible que dans la triple exclamation prêtée par al-Baydaq à Ibn Tümart au lendemain de la défaite de la Buhayra, on puisse donner au mot $a m r$ un autre sens que celui d' "Autorité». Disons tout net que le tragique de la circonstance et le ciselé des mots prononcés firent que cette exclamation put ensuite, à elle seule, suggérer qu'à côté des appellations de «calife» et d' «Amìr al-Mu'mininn», on dénommât aussi 'Abd al-Mu'min al-Amr «l'Autorité». Le Mahdī s'était écrié: «Votre Autorité est restée vivante!»... «L'Autorité reste vivante!»... «L'Autorité

59 Al-Kämil, éd. an-Nağğār, 'A. al W., Le Caire, 1356 h./1930, VIII, p. 198, 1. 26. Dans les Annales du Maghreb et de l'Espagne (Alger, 1898, p. 537) E. Fagnan a traduit: «Les dernières recommandations qu'il adressa à ses partisans furent de le prendre pour chef et d'obéir à celui qu'il appela du nom de Prince des croyants (émir el-mou'minin). Puis il mourut».

${ }^{60}$ On sait que des manuscrits sont encore retrouvés et peu à peu édités. Ainsi en est-il de deux opuscules d'Abū 1-Hasan 'Alī Ibn al-Qațtān: son Kitäb an-nazar... et son Kitāb al-iqnā $\bar{a}^{c}$ fi masā'il al-iğm $\tilde{a}^{c}$; de ce dernier, Muhammad Bencherifa a transcrit (d'après le ms.) les dernières lignes dans lesquelles l'auteur rappelle qu'il avait écrit à la demande d'Al-Manșūr et nous remarquons avec intérêt que ce calife y est désigné sous l'appellation d' al-Amr al-Hatm «l'Autorité Absolue»! (cf. Bencherifa, M., Ibn Rušd al-hafid, 1999, p. 73). 
reste vivante!» (qad baqiya Amrü-kum!... al-Amru bäqin!... al-Amru $\left.b \bar{a} q^{\text {in }} 61 !\right)$.

\begin{abstract}
ANNEXE
La façon 'id̄ārienne de désalmohadiser

Après avoir indiqué, dans la note 10 , comment Ibn ${ }^{\mathrm{c}} \mathrm{Id}$ dārī, utilisateur du Ta'rīh al-mann (T.m.) d'Ibn Șāḥib aș-Ṣalāt, avait traité les formules comportant le mot al-amr (plur. al-awāmir) utilisées dans le sens d' «ordre donné [ou reçu]» de «décisions prises», j'ai annoncé (note 18) la présente annexe dans laquelle il me paraît intéressant d'observer comment a procédé l'auteur du Bayān lorsqu'il a rencontré les multiples emplois (énumérés ci-dessus) où le mot $a l$-amr est pris dans son sens personnifié de «l'Autorité» appliqué au calife mu'minide. Pour les pages 'idāriennes concernées (B. alm., éd. Casablanca, 1985, p. 63, 1.5 à p. $126,1.6)$, la réponse est simple: pas une seule fois l'appellation n'a été acceptée! Examinons les choses de plus près.

Sur une cinquantaine de cas considérés ${ }^{62}$,

- une bonne vingtaine ont été évités, situés qu'ils sont dans des passages non «utilisés» par Ibn 'Idārī;

- dans une vingtaine d'autres, nous constatons une simple omission de la mention de «l'Autorité toute-puissante», de «la Noble-Autorité»... etc. Voici trois exemples: * ayant lu dans sa source (T.m. p. 193, 1. 11) que: «la nouvelle de ce revers (subi par le sayyid $\mathrm{Abū} \mathrm{Sa}^{\mathrm{c}} \mathrm{i} \mathrm{d}$ dans la plaine de Grenade) parvint à sa Majesté l'Autorité toute-puissante à Ribāt al-Fath près Salā [... wașala habar

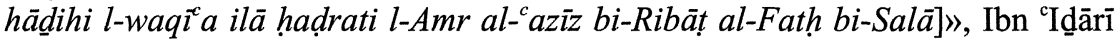
copie mais abrège en écrivant (B. alm., p. 76, 1.2-3) que: «la nouvelle de ce revers parvint à Ribāṭ al-Fath [... wașala habar hädihi-l-waqíca ilä Ribät al-Fath]»; ** ayant lu (T.m., p. 434, 1.8-9) que: les Arabes «se précipitèrent afin d'obtenir de la Noble-Autorité le pardon pour la mauvaise conduite de leurs

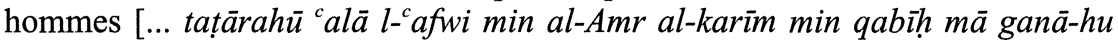
$a t b \bar{a}^{c} u$-hum]», il copie mais abrège en écrivant (B. alm., p. 117, 1. 22-23): qu' «ils se précipitèrent afin d'obtenir pardon pour la mauvaise conduite de leurs hom-

${ }^{61}$ Dans l'exclamation tūmartienne, c'est le mot $a m r$ qui a accaparé toute notre attention. Il faut néanmoins faire remarquer que le verbe baqiya et le participe actif $b \bar{a} q^{\text {in }}$ ne sont pas anodins. Bāa $q^{i n}$ est attesté dans le Qur'ān (XVI, 96): $m \bar{a}{ }^{c}{ }^{c}$ inda Allāh bāqin $" \mathrm{Ce}$ qui est en Dieu perdure!». Al-Bāqi est un des 99 noms de Dieu: «le Subsistant». On connaît d'autre part l'invocation $a b q \bar{a}-h u$ Alläh! «Que Dieu le fasse perdurer!», «Que Dieu le maintienne en vie!

${ }^{62}$ Les cas considérés sont les appellations personnifiées d'al-Amr attestées en T.m. telles qu'énumérées ci-dessus.
\end{abstract}




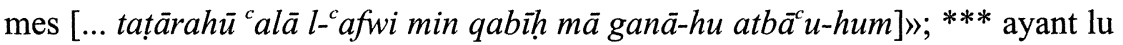
(T.m., p. 400, 1. 22): qu' «il advint par un heureux hasard dû à la baraka de cette Autorité toute-puissante que l'arrivée du sayyid coïncida avec la sortie de Firnanduh al-Bibūğ fils d'Idfunš as-Sulayțin [... wa-kāna min al-ittifāq al-hasan bi-baraka hādā l-Amr al- ${ }^{c}$ aziz an wäfaqa wușülu-hu hurūğa Firnanduh al-Bibüğ b. Idfunš as-Sulaytin]», il copie et abrège en écrivant (B. alm., p. 111, 1. 18): qu' «il advint par un heureux hasard que l'arrivée du sayyid coïncida avec la sortie d'al-Bibuğ fils d'Idfunš as-Sulayțin [... wa-kāna min al-ittifāq al-hasan ${ }^{c} a n$ wāfaqa wusūulu-hu h̆urūğa al-Bibüğ b. Idfunš as-Sulaytin]»» ${ }^{63}$.

- les cas restants (une dizaine) ont été traités par aménagement du texte: dans la phrase de sa source qu'il recopie, Ibn 'Idārī omet l'appellation personnifiée de «l'Autorité» et ensuite, soit lui substitue une appellation jugée par lui de bon aloi, soit reconstitue grammaticalement le texte:

- Dans la phrase (T.m., p. 126, 1. 3) faisant déclarer au sayyid Abū Ya quāb que le morceau poétique contenu dans la lettre arrivée d'Ifrĩqiya était «de la plume de l'Autorité toute-puissante [... min inšă' al-Amr al- $\left.{ }^{c} a z \bar{z} z\right]$ », Ibn 'Idārī écrit (B. alm., p. 65, 1.8-9): «... de la plume de son père [... min inša $\bar{a}$ ' abi-hi]]».

- Dans la phrase (T.m., p. 193, 1. 14) où il est dit qu'après le revers essuyé par le sayyid Abū Sa ${ }^{c} \overline{1} d$ dans la plaine de Grenade, «la Noble Autorité choisit parmi eux une armée énorme [... ihtära min-hum al-Amr al-karim ${ }^{c}$ askaran dah$\left.m^{a n}\right] »$, Ibn 'Id̄ārī se contente d'écrire (B. alm., p. 76, 1.4): «... il choisit parmi eux une armée énorme [... ihhtāra min-hum ${ }^{c}$ askar ${ }^{a n}$ dah̆m $\left.{ }^{a n}\right] »$.

- Dans la phrase (T.m., p. 287, 1.1) où il est dit qu'en 561 h. au retour du siège de Murcia, le sayyid Abū Sa ${ }^{c} \overline{1} d$ s'installa à Cordoue «en vertu d'un avis de l' Autorité, avis précédemment exprimé, et conformément à sa situation première [... bi-ra'y min al-Amr wa-ra'y mutaqaddim wa-ittifāq ${ }^{c}$ alā hâalati-hi al-ūlā]», Ibn 'Id̄ārī écrit (B. alm,. p. 91, 1.12-13): «... en vertu d'un avis précédemment exprimé par l' Amir et conformément à sa situation première [... bi-ra'y muqaddam min al-Amīr wa-ittifāq ${ }^{c}$ alā hạalati-hi al-ülā]».

- Dans le passage (T.m., p. 329, 1.5-6) où est évoqué le ralliement de plusieurs tribus des Șanhāğa obtenu, en 562 h. par le sayyid Abū Hafș, au lieu des mots: «... qui en fit part à l'Autorité toute-puissante [... wa-aclama al-Amr al- ${ }^{c} a z \bar{\imath} z$ bi-d̄alika]», Ibn ${ }^{c} \operatorname{Idāāi}(B . a l m .$, p. 97, 1. 6) écrit: «... qui en fit part à l'Amir [... wa-a'clama al-Amïr bi-d̄älika]».

63 Voici les références renvoyant aux autres cas identiques (on y remarquera en particulier que toutes les allusions à la baraka du calife ont été omises): (T.m., p. 116, 1.7 et $B$. alm., p. 64, 1.1); (T.m., p. 120, 1.2 et B. alm., p. 64, 1.8); (T.m., p. 130, 1.4 et B. alm., p. 66, 1.15); (T.m., p. 177, 1.2 et B. alm., p. 67, 1.23); (T.m., p. 181, 1. 6 et B. alm.; p. 73, 1.14-15); (T.m., p. 186, 1.10 et B. alm., p. 74, 1.10-11); (T.m., p. 187, 1.9 et B. alm., p. 74, 1.18); (T.m., p. 207, 1.1 et B. alm., p. 78, 1.11); (T.m., p. 294, 20 et B. alm., p. 92, 1.23); (T.m., p. 328, 1.12 et B. alm., p. 96, 1.22); (T.m., p. 369, 1.6-7 et B. alm., p. 1023); (T.m., p. 375, 1.7 et B. alm., p. $105,1.2)$; (T.m., p. $380,1.8$ et B. alm., p. $106,1.6)$; (T.m., p. $388,1.6$ et $B$. alm., p. 108 , 1.13-14); (T.m., p. 389, 1.12 et B. alm., p. 108, 1.20-21); (T.m., p. 392, 1.5 et B. alm., p. 109, 1.4-5); (T.m., p. $400,1.22$ et B. alm., p. $111,1.18)$. 
- Dans le passage (T.m., p. 339, 1.4) où l'on dit qu'en l'année 563 h., pour demander renouvellement du serment d'allégeance, «l' Autorité toute-puissante fit parvenir une noble lettre au sayyid Abū Ibrāhīm Ismā̄īl à Séville [... nafada al-Amr al- ${ }^{c}$ azīz... bi-kitāb karìm ilā as-sayyid...]», Ibn " Idārī écrit simplement (B. alm., p. 98, 1.18): «... ordre fut transmis au sayyid [... wa-nufida al-amru ilā as-sayyid]».

- Reprenant la phrase concernant Fernando Rodríguez (T.m., p. 369, 1.8) qui «s'engagea devant Dieu, sur le conseil de l' Autorité, à servir assidûment (les musulmans) [... wa- ${ }^{c} \bar{a} h a d a$ Alläh fi nașhi al-Amr bi-l-hidmati l-muğidda...]», Ibn 'Id̄ārī écrit (B. alm., p. 103, 1. 24): «il s'engagea, sur le conseil du calife, à servir assidûment (les musulmans) [...wa- ${ }^{c}$ ăhada fi nașhi al-ḥalïfa bi l-hidmati l-muğidda...]».

- A propos du même Fernando Rodríguez qui retourna dans son pays après son séjour à Marrākech en 563 h., reprenant la phrase (T.m., p. 370, 1.1): «... la Haute-Autorité ordonna qu'on lui accordât subside ainsi qu'à ses frères et compagnons [... wa-amara al-Amr al- ${ }^{c} \bar{a} l i \overline{\text {. }}$.. bi muwāsāti-hi wa-muwāsāt iȟwati-hi wa-aṣhäabi-hi]», Ibn 'Id̄ārī écrit (B. alm., p. 104, l. 1): «... et il ordonna de soutenir ses frères et ses compagnons [... wa-amara bi-ițbāt inhwati-hi wa-așhābi-hi]».

- Au sujet du ralliement d'Ibn Hamušk en 564 h., copiant la phrase (T.m., p. 390, 1.2) «... la Haute-Autorité lui répondit en lui signifiant ses sentiments de rapprochement et de conciliation [...fa-ğāwaba-hu al-Amr al- ${ }^{c} \bar{a} l i \bar{i} . . . b i$-taqrībi-hi wa-stiğlābi-hi]», Ibn 'Id̄āī écrit (B. alm., p. 108, 1.21): «... il lui répondit en lui signifiant ses sentiments de rapprochement et de conciliation [...fa-ğāwaba-hu bi-taqrībi-hi wa-stiğlābi-hi]».

- Copiant la phrase (T.m., p. 392, 1.9) où il est dit qu'en l'année 564 h., alors que le häfiz Abū Yahyā était gouverneur de Badajoz, "L'Autorité tout-puissante lui ordonna de creuser un puits à l'intérieur de la qasaba de cette place [... wa-amara-hu al-Amr al- ${ }^{c}$ aziz bi-hafr bi'r fi dāhil qașaba...]», Ibn 'Id̄āī écrit (B. alm., p. 109, 1.5-6): «Le calife se donna pour tâche de faire creuser un puits à l'intérieur de la qașaba [... wa-štaġala al-hূalīfa bi-ḥafr bi'r fī dāhil al-qașaba...]».

Dans ces neuf cas, il est clair qu' Ibn 'Id̄ārī a délibérément éliminé les utilisations mu'minides personnifiées du terme d'al-Amr «l'Autorité», soit en lui substituant une des appellations de: «son père», «l'Amir»» ou «le Calife», soit en recourant à la tournure passive ou au pronom caché «il». Nous constatons ainsi que l'appellation personnifiée d'al-Amr est absente de nos pages du Bayān ( $B$. alm., p. 63, 1.5 à p. $1261 ; 6)$ qui ont pour source le fragment retrouvé (deuxième sifr) du Ta'rīh al-mann (T.m.) d'Ibn Șāḥib aș-Ṣalāt. Est-ce à dire que cette appellation est absente du Bayān almohade tout entier?

\section{Dans le Bayān almohade tout entier}

En l'état actuel du texte édité, il apparaît — sauf inattention de notre partque le $A m r$ personnifié, absent de la soixantaine de pages examinées, l'est aussi 
des pages subséquentes jusqu'à la fin de l'ouvrage. Par contre, dans les pages antérieures - début du Bayān almohade- quelques cas de cette appellation sont attestés, appliqués à 'Abd al-Mu'min:

- en (B. alm., p. 37, 1. 9), il est rapporté, à propos de l'opposant aux almohades, Yahỵā as-Sahrāwī, qu'il se cacha, vaincu, «et se mit à supplier l'Autorité de lui pardonner et de lui accorder la vie sauve [... wa-ğa $a^{c}$ ala yataḍrra'u ilä al-Amr fì 'an yu 'fä can-hu wa-yuslama]».

- en (B. alm., p. 44, 1.16), il est rapporté que, prenant la parole au cours de la grande réunion qui se tint à Salé en 546 h., le faqih Abū Bakr Ibn al-Ğadd ${ }^{64}$, dans son discours, fit allusion aux «garçons de l'Autorité toute-puissante [... awläd al-Amr al-'aziz] ») pour désigner les fils d ${ }^{\prime} \mathrm{Abd}$ al-Mu'min dont le rôle dans le régime allait bientôt être capital.

- en (B. alm., p. 47, 1.21), il est rapporté que Yașlātan, acolyte des deux frères du Mahdi, ${ }^{\circ} \mathrm{Abd}$ al- ${ }^{\mathrm{c}} \mathrm{Azizz}$ et ${ }^{\circ} \bar{I} s a \bar{a}$, avait un comportement agressif lorsqu'il assistait au «conseil de la Haute Autorité [... mağglis al-Amr al- $\left.{ }^{-} \bar{a} l i\right]$ ]».

Ainsi donc, dans la totalité du Bayān almohade, épais de quelque 450 pages,

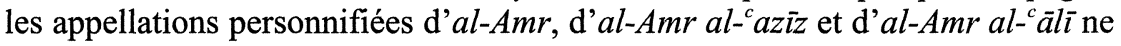
sont attestées que trois fois (une fois chacune) - sauf oubli d'inattention! - et cela dans les cinquante premières pages où elles désignent ${ }^{\mathrm{C}} \mathrm{Abd}$ al-Mu'min; dans les quatre cents pages subséquentes, «montées» à partir de sources almohades ${ }^{65}$, elles ne réapparaissent plus et ne sont jamais utilisées pour désigner chacun des onze califes suivants qui pourtant devaient en bénéficier dans les sources! Comment expliquer cette façon de faire 'idārienne? Première hypothèse, celle de l'inadvertance: Ibn 'Id̂arīi, au début de son utilisation des textes almohades, se serait laissé surprendre et aurait accueilli quelques al-Amr personnifiés avant de s'astreindre drastiquement à n'en plus admettre aucun! Seconde hypothèse, celle de la préméditation: Ibn 'Idāāî, délibérément, reconnaissant certes que ces appellations personnifiées d'al-Amr chez les mu'minides étaient abusives et qu'il allait devoir les omettre systématiquement de son texte, tint néanmoins à en retenir quelques exemples comme pour les sauvegarder «historiquement»! Pour qui l'observe tout au long de son ouvrage - surtout dans les pages où l'on peut comparer ses lignes à celles de sa source- l'auteur du Bayān, parfois maladroit dans ses réductions de textes, peut difficilement être taxé d'inadvertance. Il me semble donc que l'on doit admettre qu'ici, dans ses pages almohades, Ibn 'Iḍārī a voulu expressément «conserver» quelques unes de ces appellations contestables mais historiques d'al-Amr, tout comme il a voulu, dans

${ }^{64}$ Sur ce personnage, voir Marín, M., «Abū Bakr Ibn al-Ŷadd y su familia», dans Biografias Almohades. I, Estudios Onomástico-Biográficos de al-Andalus IX (1999), 223-59.

${ }^{65}$ Voir la liste de ces sources almohades du Bayān dans «Les țalaba dans la société almohade», p. 339 et note 31 . 
les mêmes pages, «conserver» suffisamment de talaba pour qu'on sache que leur corporation avait existé ${ }^{66}$. On sait que ces minimes entorses à la désalmohadisation peuvent être considérées comme une des causes qui valurent au Bayān sa mise à l'index en milieu mérinide; pour nous, elles sont de précieux indices de la conception que se faisait Ibn 'Idāāī de son travail d'historien. Son contemporain Ibn Abī Zar ${ }^{\mathrm{c}}$, sollicité par le palais de Fès, eut moins de scrupules en excluant radicalement des pages almohades de son Qirțās toutes les spécificités de l'époque mu'minide; l'appellation d'al-Amr en est évidemment absente. Hors du Magrib, nous l'avons dit, Ibn al-Atīir ignora vraisemblablement cet al-Amr protocolaire des mu'minides; le $\mathrm{Ka} m i l$, en tout cas, n'en a pas gardé la moindre trace. Quant au grand Ibn Haldūn, certainement fort bien renseigné sur les «originalités» almohades et mu'minides, il préféra — pour n'irriter ni les Mérinides ni les Hafṣides - un siècle après Ibn 'Id̄ārī, garder sur elles un complet silence !

\section{POST-SCRIPTUM}

\section{* Buhayra ou Bhīra?}

- Pour évoquer la bataille qui eut lieu sous les murs de Marrâkech en 524/1130, lors de laquelle les Almohades furent sévèrement battus par les Almoravides, j'ai utilisé l'appellation de «bataille de la Buhayra» adoptée communément depuis Lévi-Provençal. Après rédaction du présent article, $\mathrm{j}$ 'ai appris que cette lecture de Buhayra «pièce d'eau», «bassin» était remise en question et qu'on devait lui préférer celle de Bhìra «terre irrigée», «zone de jardins». [Voir sur ce sujet: "Itinéraire culturel des Almoravides et des Almohaces - Maghreb et Péninsule ibérique», pp. 72-76. - M. Rabitat al-Din: «Al-Bhira» dan Ma 'lamat al-Magrib t. II, pp. 1085-1088. Consulter aussi: A. L. de Premare «Dictionnaire Arabe-Français - Langue et culture marocaines - t. I, p. 144 ].

\section{RESUMEN}

La palabra amr («orden dada» - «autoridad») vino a ser, entre los Almohades Mu'miníes, una denominación utilizada para designar a 'Abd al-Mu'min y a cada uno de sus sucesores los califas: «al-Amr» («la Autoridad»), «hādā l-Amr» («esta Autoridad»).

Este artículo propone como origen de este uso mu'miní el recuerdo de las exclamaciones atribuidas al mahdī Ibn Tūmart, en las cuales había designado a ${ }^{\text {c} A b d ~ a l-M u ' m i n ~ c o m o ~ e l ~ f u t u r o ~ p o s e e d o r ~ d e l ~ a m r ; ~ e n t r e ~ e s t a s ~ e x c l a m a c i o n e s ~}$ tūmartíes se debe dar un lugar de preferencia a la que - entendida de otra mane-

${ }^{66}$ Cf. «Les țalaba dans la société almohade», p. 340. 
ra hasta ahora- había proferido poco tiempo antes de su muerte, al enterarse de la derrota almohade de la Buhayra.

\section{RÉSUMÉ}

Le mot amr («ordre donné» - «autorité») devint, chez les Almohades Mu'minides, une appellation utilisée pour désigner 'Abd al-Mu'min et chacun de ses successeurs: «al-Amr» («l'Autorité»), «hā

Le présent article propose que l'on donne pour origine à cet usage Mu'minide le souvenir des exclamations prêtées au mahdī Ibn Tūmart dans lesquelles il avait désigné 'Abd al-Mu'min comme le futur détenteur du amr; parmi ces exclamations tūmartiennes, une place spéciale doit être faite à celle - comprise autrement jusqu'à présent- qu'il avait proférée, peu de temps avant sa mort, en apprenant la défaite almohade de la Buhayra. 